\title{
Gauged Neural Network: Phase Structure, Learning, and Associative Memory
}

\author{
Motohiro Kemuriyama and Tetsuo Matsui \\ Department of Physics, Kinki University, Higashi-Osaka, 577-8502 Japan \\ Kazuhiko Sakakibara \\ Department of Physics, Nara National College of Technology, Yamatokohriyama, 639-1080 Japan,

\begin{abstract}
A gauge model of neural network is introduced, which resembles the $\mathrm{Z}(2)$ Higgs lattice gauge theory of high-energy physics. It contains a neuron variable $S_{x}= \pm 1$ on each site $x$ of a $3 \mathrm{D}$ lattice and a synaptic-connection variable $J_{x \mu}= \pm 1$ on each link $(x, x+\hat{\mu})(\mu=1,2,3)$. The model is regarded as a generalization of the Hopfield model of associative memory to a model of learning by converting the synaptic weight between $x$ and $x+\hat{\mu}$ to a dynamical $\mathrm{Z}(2)$ gauge variable $J_{x \mu}$. The local $\mathrm{Z}(2)$ gauge symmetry is inherited from the Hopfield model and assures us the locality of time evolutions of $S_{x}$ and $J_{x \mu}$ and a generalized Hebbian learning rule. At finite "temperatures", numerical simulations show that the model exhibits the Higgs, confinement, and Coulomb phases. We simulate dynamical processes of learning a pattern of $S_{x}$ and recalling it, and classify the parameter space according to the performance. At some parameter regions, stable column-layer structures in signal propagations are spontaneously generated. Mutual interactions between $S_{x}$ and $J_{x \mu}$ induce partial memory loss as expected.
\end{abstract}

\section{INTRODUCTION}

Our brains exhibit various complicated functions like recognition, thinking, etc. In particular, it is quite interesting to understand the mechanism of storing and restoring particular concepts, i.e., how to learn and recall them. It is challenging to construct a mathematical model to describe the essence of learning and recalling.

In the framework of neural network, it is well known that the Hopfield model [1] of associative memory offers us a reasonable mechanism how to recall the images and patterns that one has once learned. In the Hopfield model, the state of the $i$-th neuron, excited or unexcited, is described by the $\mathrm{Z}(2)$ variable $S_{i}(=$ $\pm 1)(i=1,2, \ldots, N)$, and the state of the synaptic connection between the $i$-th and $j$-th neurons is expressed by its strength (weight), $J_{i j}$, to transmit signals, which is a preassigned real constant. The signal at the $j$-th site at time $t$ propagates to the $i$-th site through the axon and the synaptic connection in the form $J_{i j} S_{j}(t)$ to affect the state $S_{i}$ in the next time step $t+\epsilon$ as

$$
S_{i}(t+\epsilon)=\operatorname{sgn}\left[\sum_{j=1}^{N} J_{i j} S_{j}(t)\right] .
$$

Physically, $S_{j}$ is the signature of the electric potential at the $j$-th neuron (membrane potential) measured from a certain common level, and $J_{i j}$ is the conversion factor to propagate the potential from $j$ to $i$. So the quantity $\sum_{j} J_{i j} S_{j}$ in the bracket is just the total amount of potential accumulated at the $i$-th neuron. The case $J_{i j}>0$ enhances the tendency that the $i$-th neuron shall take the same state as that at $j, S_{i}=S_{j}$, while the case $J_{i j}<0$ suppresses it, favoring the opposite state, $S_{i}=-S_{j}$.

The time evolution (1.1), when applied to all the variables $S_{i}$ in an asynchronous manner, is known to decrease (not increase) the following "energy",

$$
E=-\frac{1}{2} \sum_{i, j=1}^{N} S_{i} J_{i j} S_{j}
$$

Let us prepare $M$ patterns $S_{i}=\xi_{i}^{\alpha}(\alpha=1,2, \ldots, M)$ (which are mutually orthogonal, $\sum_{i} \xi_{i}^{\alpha} \xi_{i}^{\beta}=N \delta_{\alpha \beta}$ ) as the patterns to learn. One may choose $J_{i j}$ so that each of these patterns, $S_{i}=\xi_{i}^{\alpha}$, is a local minimum of this energy. Such an idea is realized by taking the following Hebbian learning rule [2];

$$
J_{i j}=\frac{1}{M} \sum_{\alpha=1}^{M} \xi_{i}^{\alpha} \xi_{j}^{\alpha} .
$$

If we start from a pattern of $S_{i}(0)$, then one of the memorized patterns that locates nearest to $S_{i}(0)$, say $\xi_{i}^{\alpha}$, is obtained gradually via the rule $(1.1), S_{i}(t)=\xi_{i}^{\alpha}$ in a certain time [3]. This certainly offers us a possible mechanism of associative memory.

How about processes of learning patterns? The Hopfield model itself is incapable for this purpose, since the memorized patterns are stored in the fixed parameters $J_{i j}$ as (1.3) from the beginning. We need some generalization so that these $J_{i j}$ change as time goes by, i.e., plasticity of $J_{i j}$. A simple example of the time dependence of $J_{i j}$ is the following;

$$
J_{i j}(t+\epsilon)=J_{i j}(t)+\frac{1}{M} \xi_{i}^{\alpha} \xi_{j}^{\alpha} .
$$

It reflects the Hebbian rule (1.3), describing the process that the pattern $S_{i}=\xi_{i}^{\alpha}$ is learned in the period $(t, t+\epsilon)$.

A well-known model of learning is a perceptron or its improvement, the error back-propagation model [4]. In 
a perceptron, one assigns a pattern $S_{i}=\xi_{i}$ for neurons $S_{i}$ in the input layer and optimizes the weight $w_{i}$ connecting $S_{i}$ and the single output neuron $S^{\prime}$ so that $S^{\prime} \equiv \theta\left(\sum_{i} w_{i} S_{i}\right)$ produces $1(0)$ for $S_{i}=\xi_{i}\left(S_{i} \neq \xi_{i}\right)$ approximately. The model proves itself very useful for the problems of pattern recognition. However, in a perceptron, (i) the flow of informations is one way from the input layer to the output neuron, and (ii) the time evolution of $S_{i}(t)$ is missing, although the process of optimization of $w_{i}$ itself may be regarded as a time evolution of $J_{i j}$. These two points seem quite different from what is going on in our brains.

In a set of models of self-organization [5-8], signals from the neurons $S_{j}$ in the input layer are forwarded with the weights $w_{i j}$ to the neurons $S_{i}^{\prime}$ in the output layer, and $w_{i j}(t)$ are assumed to evolve with the rule like $w_{i j}(t+\epsilon)-w_{i j}(t)=-\alpha w_{i j}+\beta S_{i}^{\prime} S_{j}$, i.e., with the damping effect and the Hebbian rule (and/or certain constraints on $w_{i j}$ ). It is shown that the neurons in the output layer are partitioned into subsets, and the neurons in each subset become active for a particular pattern in the input layer. This may explain the emergence of socalled column structures of active neurons observed in human brains like ocular dominance columns, orientation columns, and direction columns in the medial temporal area. However, these models also share the properties (i) and (ii) above.

As a stochastic model of learning, Boltzmann machines [9] are well known. Here $S_{i}$ develops according to Boltz-

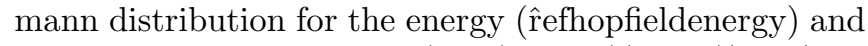
the learning rule is like $w_{i j}(t+\epsilon)-w_{i j}(t)=\beta\left(\left\langle S_{i} S_{j}\right\rangle_{\xi}-\right.$ $\left.\left\langle S_{i} S_{j}\right\rangle_{E}\right)$, where the first term in R.H.S. is an average over patterns to learn and describes the Hebbian rule whereas the second term is an average over Bolotzmann distribution and describes so-called unlearning processes.

Following these pioneering works, various models of learning have appeared. Some models treat both $S_{i}(=$ $\pm 1)$ and $J_{i j}[\in(-\infty, \infty)]$ as dynamical variables [10]. Although they contain certain interesting features, it seems necessary to consider some natural and explicit principles that characterize the dynamics of $S_{i}$ and $J_{i j}$.

In Ref. [11], a gauge model of neural network is proposed. Here $J_{i j}$ are regarded as gauge variables, i.e., path-dependent phase factors (exponentiated gauge field) and the energy of the model is obtained by adding a couple of additional gauge-invariant terms to the Hopfield energy (1.2). Both $S_{i}$ and $J_{i j}$ are treated as dynamical variables and their time-dependences at finite "temperature" $(T)$ are postulated to obey a stochastic process for Boltzmann distribution of the gauge-invariant energy. The model on a $3 \mathrm{D}$ lattice at finite $T$ resembles the lattice gauge theory [12] in high-energy particle physics. The phase structure of lattice gauge theory was intensively examined by mean field theory (MFT) and Monte Carlo (MC) simulations [13]. Generally, three phases, Higgs phase, Coulomb phase, and confinement phase, are known to be possible [see the table (4.7) below]. In the gauge model of neural network, the former two corre- spond to the ferromagnetic phase $\left(\left\langle J_{i j}\right\rangle \neq 0,\left\langle S_{i}\right\rangle \neq 0\right.$ in MFT $)$ and the paramagnetic phase $\left(\left\langle J_{i j}\right\rangle \neq 0,\left\langle S_{i}\right\rangle=0\right)$ in the Hopfield model, respectively. The third confinement phase has $\left\langle J_{i j}\right\rangle=0$ and $\left\langle S_{i}\right\rangle=0$, describing the state that both learning and recalling are disable. For example, the Higgs phase corresponds to a smart student who took a lesson and made a good score in examination; the Coulomb phase to a student who took a lesson but with a bad score; the confinement phase to a student who cuts a class.

There is a quite interesting relation between the present gauge model and the quantum memory of a toric code studied by Kitaev et al. [14]. To calculate the accuracy threshold of a $2 \mathrm{D}$ toric quantum memory, one needs to study the phase diagram of the $3 \mathrm{D}$ pure $\mathrm{Z}(2)$ lattice gauge theory with random gauge coupling. The present model at $c_{1}=c_{3}=0$ (see Sect.IIIA) coincides this gauge model at vanishing randomness. The error-free condition in uploading data to and downloading from a quantum memory leads us that this pure $\mathrm{Z}(2)$ gauge model should be in the ordered (Coulomb) phase [15]. Thus the ability of leaning patterns in the gauged neural network corresponds to the error-free function of a quantum memory of a toric code.

In this paper, we study various aspects of this $\mathrm{Z}(2)$ gauged neural network in detail. We investigate the full phase structure of the system at finite $T$. We also simulate the dynamical processes of learning a pattern of $S_{i}$ and recalling it, and examine the parameter dependence of the rate of performance.

Here we note that Thoulouse [16] noticed Z(2) gauge symmetry in the related models, the models of spin glasses [17], and adopted it to characterize effects of frustration. In fact, the Sherrington-Kirkpatrick model of spin glasses [17] has the same form of energy as that of the Hopfield model (1.2). Since then, there have appeared various studies on frustrations in spin glasses [18]. The crucial difference between the models of spin glasses and the present model of neural network is in that the "gauge variables" $J_{i j}$ are regarded as quenched random variables in the spin-glass theory whereas they are treated in the present neural network as dynamical variables on an equal footing to the dynamical neuron variables $S_{i}$.

The structure of the paper is as follows; In Sect.II, we explain the origin and the relevance of $\mathrm{Z}(2)$ gauge symmetry in neural networks in details. In Sect.III, we introduce the $\mathrm{Z}(2)$ gauge model on a 3D lattice. In Sect.IV, we study its phase structure at finite $T$ by statistical mechanics and find emergent column-layer structures. In Sect.V, we simulate dynamical processes of learning a pattern and recalling it. In Sect.VI, we present discussion and the problems in future. 


\section{Z(2) GAUGE SYMMETRY}

In this section, we explain the origin of the gauge symmetry and its relevance to neural networks.

Let us start with the Hopfield model (1.2). Here one may assign $S_{i}=1$ for the excited (fired) state of the $i$-th neuron and $S_{i}=-1$ for the unexcited state. Let us focus on the part of network consisting of the $j$-th neuron and the axon and synaptic connection starting from the $j$-th neuron and ending at the $i$-th neuron, and consider the following two states (a) and (b) of this part;

$$
\begin{array}{ll}
\text { state (a): } & \left(S_{j}, J_{i j}\right), \\
\text { state (b) : } \quad\left(S_{j}^{\prime}=-S_{j}, J_{i j}^{\prime}=-J_{i j}\right) .
\end{array}
$$

Physically, they are independent(different) each other. For example, (a) $\left(S_{j}=1, J_{i j}=J>0\right)$ describes the excited neuron and the enhancing connection, while (b) $\left(S_{j}=-1, J_{i j}=-J<0\right)$ describes the unexcited neuron and the suppressing (inhibitory) connection. In the Hopfield model, these two states (a) and (b) are degenerate; they have the same amount of energy (1.2). Actually, the energy $E_{i j}$ stored in the above part is

$$
\begin{aligned}
E_{i j}(b) & \equiv-S_{i} J_{i j}^{\prime} S_{j}^{\prime}=-S_{i}\left(-J_{i j}\right)\left(-S_{j}\right) \\
& =-S_{i} J_{i j} S_{j}=E_{i j}(a) .
\end{aligned}
$$

The state (b) is obtained from (a) by the following replacement of $S_{j}$ and $J_{i j}$;

$$
S_{j} \rightarrow S_{j}^{\prime} \equiv V_{j} S_{j}, \quad J_{i j} \rightarrow J_{i j}^{\prime} \equiv J_{i j} V_{j}, \quad V_{j}=-1 .
$$

In lattice gauge theory [12], the replacement (2.3) is known as a local $\mathrm{Z}(2)$ gauge transformation in which $J_{i j}$ plays the role of a so-called gauge variable. More generally, one may consider the following general local (site-dependent) $\mathrm{Z}(2)$ gauge transformation throughout the network (for all $i$ and $j$ );

$$
S_{i} \rightarrow S_{i}^{\prime} \equiv V_{i} S_{i}, \quad J_{i j} \rightarrow J_{i j}^{\prime} \equiv V_{i} J_{i j} V_{j}, \quad V_{i}= \pm 1 .
$$

Here, whether one replaces the state (a) by (b) $\left(V_{i}=-1\right)$ or not $\left(V_{i}=1\right)$ may depend site by site. It is easy to see that the Hopfield energy (1.2) is invariant under this transformation.

We regard this "gauge symmetry" of the Hopfield model as an important property that the generalized model of learning should inherit. To generalize the Hopfield model to such a gauge model of learning, we just need (i) to convert $J_{i j}$ from constants to dynamical (gauge) variables, which are to transform as (2.4), and (ii) to introduce a gauge-invariant energy.

In Fig.1 we illustrate the function of the gauge field $A_{\mu}(x)$ in the conventional $\mathrm{U}(1)$ gauge field theory by using the path-dependent phase factor,

$$
U(x, y) \equiv \exp \left(i \int_{P_{x y}} d x_{\mu}^{\prime} A_{\mu}\left(x^{\prime}\right)\right),
$$

where $P_{x y}$ is a certain path connecting two spatial points $y$ and $x$. It conveys informations about the relative orientation of two internal coordinates at $x$ and $y$, and parallel-translates a vector $\varphi(y)$ at $y$ to $x$ along a path from $y$ to $x$, giving rise to a vector $U(x, y) \varphi(y)$ at $x$.

Since the strength $J_{i j}$ of the synaptic connection describes the way how the electric signal at $j$ is transformed to $i$, it is natural also from this gauge-theoretical point of view to identify $J_{i j}$ as $U(x, y)$ in gauge theory. Then the resulting signal conveyed from $j$ to $i, J_{i j} S_{j}$, corresponds to $U(x, y) \varphi(y)$.

Let us explain the relation between the gauge symmetry and the physical states in detail [20]. One may first think that the assignment of the gauge-variant variable $S_{i}= \pm 1$ to the the physical (excited and unexcited) states is inappropriate, because a gauge transformation $S_{i}^{\prime}=-S_{i}$ exchanges the excited state and the unexcited state. However, such an assignment brings no difficulties because $J_{i j}$ also changes. Let us start with the global frame of $S_{i}$ illustrated in Fig.2a, where $S_{i}=1(-1)$ describe the excited(unexcited) state of the $i$-th neuron for all $i$. Suppose that we make a local gauge transformation with $V_{i}=1-2 \delta_{i j}$, which implies that $S_{j}^{\prime}=1(-1)$ describes the unexcited(excited) state. See Fig.2b. Such a capricious or perverse choice is "corrected" or "compensated" by the associated transformation $J_{i j}^{\prime}=-J_{i j}$, thus bringing no changes in physical content. Actually, the state at $j$ is to be recognized at the neighboring observer at $i$ by the combination $J_{i j} S_{j}$. There holds the equality $J_{i j} S_{j}=J_{i j}^{\prime} S_{j}^{\prime}$, which shows that the physical state at $j$ does not change.

From these considerations, we regard $J_{i j}$ as the pathdependent phase factors of the gauge group $\mathrm{Z}(2)$, hence they take $\mathrm{Z}(2)$ variables,

$$
J_{i j}= \pm 1 \in Z(2)
$$

in appropriate unit (which is to be supplied through the coefficients in the energy). One may claim that $J_{i j}$ should take continuous real values $-\infty<J_{i j}<\infty$ instead of $\mathrm{Z}(2)$ variables. Such an assignment is certainly possible in constructing a gauge model, as long as $J_{i j}$ transform as (2.4). However, there should be certain constraints on $J_{i j}$, which reflect, e.g., conservation laws of chemical materials and restrict their possible values. On this point, study of the biological models of self-organization [5-8] is suggestive and can be taken as a support to our simplification (2.6). For example, von der Malsburg [6] puts the constraint $\sum_{j} J_{i j}=1$ for each $i$, while Tanaka et al. [7] argue that the values of $J_{i j}$ can be well approximated by the Potts spin variables; $J_{i j}=0$ or $1, \sum_{j} J_{i j}=1$. While both authors focus on the enhancing connections $\left(J_{i j}>0\right)$, Linsker [8] considers also the case of suppressing connections $\left(J_{i j}<0\right)$ and puts the constraints $-1<J_{i j}<1$. Our treatment $(2.6)$ may be viewed (i) as the special case of Linsker's treatment that most configurations are to reach the end points of his inequality, and/or (ii) as a generalization of Tanaka's result to the 
$\mathrm{Z}(2)$ gauge theory in which both $J_{i j}>0$ and $J_{i j}<0$ should appear as (2.4) indicates [19].

Concerning to the signature of $J_{i j}$. it was once thought that each neuron releases only one type of neurotransmitter (Dale's law), which implies that the signature of $J_{i j}$ should be fixed for each $j$. However, there is now evidence that Dale's law does not hold, indicating neurons release more than one kind of neurotransmitter. Irrespective of experimental circumstances including this case, we have a sound reason to consider $J_{i j}$ with indefinite signature. It is supplied by regarding the gauge model under consideration as an effective model of renormalization-group theory as explained in Sect.IIIA for the explicit lattice model. Each $S_{i}$ is associated there not with a single neuron but with a cluster of neurons, expressing the "average" over the states of neurons contained in each cluster. $J_{i j}$ is also the average strength between two clusters, so its signature is to be indefinite.

As stated before, the $\mathrm{Z}(2)$ gauge symmetry requires that the energy $E\left(\left\{S_{i}\right\},\left\{J_{i j}\right\}\right)$ of the system is gaugeinvariant;

$$
\begin{aligned}
S_{i}(= \pm 1) & \rightarrow S_{i}^{\prime} \equiv V_{i} S_{i}, \\
J_{i j}(= \pm 1) & \rightarrow J_{i j}^{\prime} \equiv V_{i} J_{i j} V_{j}, \quad V_{i}= \pm 1, \\
E\left(\left\{S_{i}^{\prime}\right\},\left\{J_{i j}^{\prime}\right\}\right) & =E\left(\left\{S_{i}\right\},\left\{J_{i j}\right\}\right) .
\end{aligned}
$$

The principle of gauge symmetry puts severe restrictions on the form of $E$. Some possible gauge-invariant terms are depicted in Fig.3. The first term $S_{i} J_{i j} S_{j}$ in Fig.3 is just the term of the Hopfield model. The second term $S_{i} J_{i k} J_{k j} S_{j}$ may be viewed to describe the combined effect of the two successive processes $S_{k} J_{k j} S_{j}$ and $S_{i} J_{i k} S_{k}$. Actually, due to $S_{k}^{2}=1$, we have

$$
S_{i} J_{i k} S_{k} \times S_{k} J_{k j} S_{j}=S_{i} J_{i k} J_{k j} S_{j} .
$$

In the same way, the last two terms are interpreted as

$$
\begin{aligned}
J_{i j} J_{j i} & =S_{i} J_{i j} S_{j} \times S_{j} J_{j i} S_{i}, \\
J_{i j} J_{j k} J_{k i} & =S_{i} J_{i j} S_{j} \times S_{j} J_{j k} S_{k} \times S_{k} J_{k i} S_{i} .
\end{aligned}
$$

These two terms describe the combined processes taking place along closed circuits. Hebb [2] has once introduced the concept of so called reverberating circuits, in which short-term memories are considered to be stored. These terms (2.9) may describe functions of such circuits.

\section{MODEL}

\section{A. $Z(2)$ gauge theory on a $3 \mathrm{D}$ lattice}

To be explicit, let us formulate the gauge model on a $3 \mathrm{D}$ cubic lattice. Then the system resembles to the lattice gauge theory introduced by Wilson [12], and known ample techniques are applicable to study it. We specify each site by the site-index $x$ and use $\mu=1,2,3$ as the direction index. We use $\mu$ also as the unit vector in the $\mu$-th direction. We set the lattice spacing $a=1$ for simplicity. For each $x$ we put a $\mathrm{Z}(2)$ spin variable,

$$
S_{x}= \pm 1
$$

as $S_{i}$ with $i \leftrightarrow x$, and for each link $(x \mu) \equiv(x, x+\mu)$, i.e., for nearest-neighbor $(\mathrm{NN})$ pair of sites, we put another $\mathrm{Z}(2)$ variable,

$$
J_{x \mu}= \pm 1
$$

as $J_{i j}$ with $x \leftrightarrow j, x+\mu \leftrightarrow i$. Below we consider the symmetric connections, i.e., $J_{i j}=J_{j i}$, so $J_{x \mu}$ describes also the conductivity of signals propagating in the opposite direction from $x+\mu$ to $x$. $J_{x \mu}$ is the path-dependent phase factor of gauge theory as discussed in the previous section. The local $\mathrm{Z}(2)$ gauge transformation is given by

$$
\begin{aligned}
S_{x} & \rightarrow S_{x}^{\prime} \equiv V_{x} S_{x}, \\
J_{x \mu} & \rightarrow J_{x \mu}^{\prime} \equiv V_{x+\mu} J_{x \mu} V_{x}, \\
V_{x} & = \pm 1 .
\end{aligned}
$$

As the energy $E$ of the system, we propose

$$
\begin{aligned}
E= & -c_{1} \sum_{x} \sum_{\mu} S_{x+\mu} J_{x \mu} S_{x} \\
& -c_{2} \sum_{x} \sum_{\mu>\nu} J_{x \mu} J_{x+\mu, \nu} J_{x+\nu, \mu} J_{x \nu} \\
& -c_{3} \sum_{x} \sum_{\mu} \sum_{\nu(\neq \mu)}\left(S_{x} J_{x \nu} J_{x+\nu, \mu} J_{x+\mu, \nu} S_{x+\mu}\right. \\
& \left.+S_{x} J_{x-\nu, \nu} J_{x-\nu, \mu} J_{x-\nu+\mu, \nu} S_{x+\mu}\right) .
\end{aligned}
$$

Each term in (3.4) is depicted in Fig.4. The term $c_{1}$, which corresponds to the energy of the Hopfield model, describes the direct transfer of signals from $x$ to $x+\mu$. The term $c_{2}$ describes the self-energy of transfer of signal through the contour $(x \rightarrow x+\mu \rightarrow x+\mu+\nu \rightarrow x+$ $\nu \rightarrow x$ ) and the contour with the opposite direction as a reverberating circuit (2.9). The term $c_{3}$ describes indirect transfers of signal from $x$ to $x+\mu$ via the bypath, $(x \rightarrow$ $x+\nu \rightarrow x+\nu+\mu \rightarrow x)$ as explained in(2.8). Each term of $E$ is gauge invariant, so

$$
E\left(\left\{S_{x}^{\prime}\right\},\left\{J_{x \mu}^{\prime}\right\}\right)=E\left(\left\{S_{x}\right\},\left\{J_{x \mu}\right\}\right) .
$$

Appearance of the reverberating $c_{2}$ and indirect $c_{3}$ terms is also supported by the renormalization theory of critical phenomena. After a renormalizationgroup transformation to coarse-grain the system in the space and/or time scales by integrating out the shortwave-length/high-frequency modes of variables, every term in the energy of the resulting effective theory acquires corrections due to the integrated variables. These renormalization-group transformations also generate terms that are not contained in the original energy. In our case, if one starts with only the $c_{1}$-term, the $c_{2}$ and $c_{3}$ terms, among other terms, are certainly generated as 
these effective interaction terms. We note that these effective terms are necessarily Z(2) gauge-invariant reflecting the gauge symmetry of the $c_{1}$ term. This supports our postulate that the generalized model should respect the local $\mathrm{Z}(2)$ gauge symmetry that has been revealed in the Hopfield model.

As stated in Sect.I, the present system is similar to the $\mathrm{Z}(2)$ lattice gauge theory [12]. There, $S_{x}$ is interpreted as a Higgs (matter) field and $J_{x \mu}$ is the exponentiated gauge field, $J_{x \mu}=\exp \left(i A_{x \mu}\right)$. Its standard energy (action) is given by the first two $\left(c_{1}\right.$ and $\left.c_{2}\right)$ terms; the $c_{1}$ term represents the kinetic energy of Higgs particles interacting with the gauge field $A_{x \mu}$, while the $c_{2}$ term represents the energy of the gauge field. Actually, in the electromagnetic $U(1)$ gauge theory, this $c_{2}$ term represents the energy density $\vec{E}^{2}+\vec{B}^{2}$ of the electromagnetic field [12]. The values of these coefficients $c_{i}$ are related in principle with the energy of the original model that is defined at the microscopic level. This microscopic model is, however, not easy to specify, so we regard these $c_{i}$ as effective parameters in a phenomenological point of view or in the sense of renormalization-group in the following discussion. This set of parameters may differ individually, characterizing each neural network, i.e., each brain.

\section{B. Time evolution}

Let us consider the dynamics of $S_{x}(t)$ and $J_{x \mu}(t)$. We postulate that the energy $E$ basically decreases as the time increases, which sounds natural since our brain is a dissipative system (as long as $E$ is regarded as the physical energy). This rule of energy decrease may fail with some rates due to misfunctions of signal processing caused by noises, etc., and may be controlled by a parameter which we call "temperature" $T \in(0, \infty)$; For higher(lower) $T$, failures occur more(less). This $T$ should not be confused with the physical temperature of brain, although there may be some correlations among them.

For a system of continuous variables, these postulates are implemented in Langevin equations of time evolution [21]. For discrete $\mathrm{Z}(2)$ variables like $S_{x}, J_{x \mu}$, we propose to use the Metropolis algorithm (MA) [22] as the rule of time evolutions. In fact, MA was adopted to study the dynamics of the Hopfield model in the name of Boltzmann machine [9]. MA is a standard algorithm to calculate the thermal averages $\langle O\rangle$ over Boltzmann distribution with the energy $E$,

$$
\begin{aligned}
\langle O(S, J)\rangle & =\frac{1}{Z} \sum_{S, J} O(S, J) \exp (-\beta E(S, J)), \beta \equiv \frac{1}{T} \\
Z & =\sum_{S, J} \exp (-\beta E(S, J))
\end{aligned}
$$

By starting with a certain initial state $\left\{S_{x}(0), J_{x \mu}(0)\right\}$, MA determines $\left\{S_{x}(\ell+1), J_{x \mu}(\ell+1)\right\}$ from $\left\{S_{x}(\ell), J_{x \mu}(\ell)\right\}$ by a certain probabilistic rule, and generates a Markov(stochastic) process,

$$
\left\{S_{x}(0), J_{x \mu}(0)\right\},\left\{S_{x}(1), J_{x \mu}(1)\right\},\left\{S_{x}(2), J_{x \mu}(2)\right\}, \cdots
$$

In Fig.5, we present a flow chart of the MA for a system of $\mathrm{Z}(2)$ variables $S$. Then it is assured [22] that the following relation holds;

$$
\langle O(S, J)\rangle=\lim _{M \rightarrow \infty} \frac{1}{M} \sum_{\ell=1}^{M} O\left(\left\{S_{x}(\ell), J_{x \mu}(\ell)\right\}\right) .
$$

We regard the Markov process (3.7) just as the time evolutions of $S_{x}(t)$ and $J_{x \mu}(t)$ from $t$ to $t+\epsilon(\epsilon$ is a certain time interval) with the relation $t=\epsilon \ell$. Then the relation (3.8) means that our time evolution converges at large time to the equilibrium distribution given by (3.6). The time interval $\epsilon$ is related with the MA parameters $\alpha_{S}, \alpha_{J}$ (written as $\alpha$ in Fig.5), which control the rates of changes from $S_{x}(\ell)$ to $S_{x}(\ell+1)$ and from $J_{x \mu}(\ell)$ to $J_{x \mu}(\ell+1)$, respectively; $\alpha \rightarrow 1(0)$ implies slow(fast) changes.

As Fig. 5 shows, $S_{x}(t+\epsilon)$ and $J_{x \mu}(t+\epsilon)$ are determined by the energy difference like $\Delta E=E\left(-S_{x}(t)\right)-$ $E\left(S_{x}(t)\right) \propto \partial E / \partial S_{x}$ and $\Delta E=E\left(-J_{x \mu}(t)\right)-$ $E\left(J_{x \mu}(t)\right) \propto \partial E / \partial J_{x \mu}$. These partial derivatives [23] are given by

$$
\begin{aligned}
\frac{\partial E}{\partial S_{x}} & =-c_{1} \sum_{\mu}\left(S_{x+\mu} J_{x \mu}+S_{x-\mu} J_{x-\mu, \mu}\right) \\
& -c_{3} \sum_{\mu} \sum_{\nu(\neq \mu)}\left(J_{x \nu} J_{x+\nu, \mu} J_{x+\mu, \nu} S_{x+\mu}\right. \\
& +J_{x-\nu, \nu} J_{x-\nu, \mu} J_{x-\nu+\mu, \nu} S_{x+\mu} \\
& +S_{x-\mu} J_{x-\mu, \nu} J_{x+\nu-\mu, \mu} J_{x \nu} \\
& \left.+S_{x-\mu} J_{x-\nu-\mu, \nu} J_{x-\nu-\mu, \mu} J_{x-\nu, \nu}\right), \\
\frac{\partial E}{\partial J_{x \mu}} & =-c_{1} S_{x+\mu} S_{x} \\
& -c_{2} \sum_{\nu(\neq \mu)}\left(J_{x+\mu, \nu} J_{x+\nu, \mu} J_{x \nu}+J_{x-\nu+\mu, \nu} J_{x \mu} J_{x-\nu, \nu}\right) \\
& -c_{3} \sum_{\nu(\neq \mu)}\left(S_{x-\nu} J_{x-\nu, \nu} J_{x+\mu-\nu, \nu} S_{x+\mu-\nu}\right. \\
& +S_{x+\nu} J_{x \nu} J_{x+\mu, \nu} S_{x+\mu+\nu}+S_{x} J_{x+\mu, \nu} J_{x+\nu, \mu} S_{x+\nu} \\
& +S_{x} J_{x+\mu-\nu, \nu} J_{x-\nu, \mu} S_{x-\nu}+S_{x+\nu+\mu} J_{x+\nu, \mu} J_{x \nu} S_{x+\mu} \\
& \left.+S_{x-\nu+\mu} J_{x-\nu, \mu} J_{x-\nu, \nu} S_{x+\mu}\right) .
\end{aligned}
$$

From these expressions, we observe a couple of merits of the gauge-invariant energy;

(i) The time evolution of $S_{x}$ is affected only through the terms containing $J_{x \mu}$ and $J_{x-\mu, \mu}$ that have contacts with $S_{x}$ by inspecting $\partial E / \partial S_{x}$ Likewise, the evolution of $J_{x \mu}$ is driven by the terms that have contact points $x$ and $x+\mu$ as one inspects $\partial E / \partial J_{x \mu}$. The gauge symmetry assures us that the time evolutions take place through local interactions. As stated in Sect.II, this is a welcome property. 
(ii) The first term in the right-hand-side of the second equation of (3.9) expresses just the Hebbian rule (1.3) to learn the present pattern $S_{x}$ for $c_{1}>0$. The remaining terms in the right-hand-side describe the indirect effects by neighboring $S_{x}$ and $J_{x \mu}$, which generalize the Hebbian rule.

The typical time scales of variations in $S_{x}(t)$ and $J_{x \mu}(t)$ may be different in general. Actually, it is widely accepted that, in the actual processes occurring in human brain, the changes in $J_{i j}$ is much slower than those of $S_{i}$. One may take it into account by controlling the characteristic time scales of these two sets of variables by assigning different values for the parameters as $\alpha_{S}<\alpha_{J}$. We come back to this point in Sect.V.

\section{PHASE STRUCTURE}

In this section, we study the phase structure of the system with the energy (3.4) at finite $T$ by using MA (3.7). In Sect.IVA, we formulate the MFT and present the resulting phase diagram together with the results of MC simulations. Some special cases of the model are also discussed. In Sect.IVB, we explain the details of MC simulations with MA. In Sect.IVC, we study the columnlayer structure for $c_{2}<0$.

\section{A. Mean field theory and the phase diagrams}

The MFT may be formulated as a variational method [24] for the Helmholtz free energy $F$;

$$
Z=\prod_{x} \sum_{S_{x}= \pm 1} \prod_{x, \mu} \sum_{J_{x \mu}= \pm 1} \exp (-\beta E) \equiv \exp (-\beta F) .
$$

Actually, for a trial energy $E_{0}$ there holds the following relations;

$$
\begin{aligned}
Z_{0} & =\prod_{x} \sum_{S_{x}= \pm 1} \prod_{x, \mu} \sum_{J_{x \mu}= \pm 1} \exp \left(-\beta E_{0}\right) \equiv \exp \left(-\beta F_{0}\right) \\
\langle O\rangle_{0} & \equiv Z_{0}^{-1} \prod_{x} \sum_{S_{x}= \pm 1} \prod_{x, \mu} \sum_{J_{x \mu}= \pm 1} O \exp \left(-\beta E_{0}\right) \\
F & \leq F_{v} \equiv F_{0}+\left\langle E-E_{0}\right\rangle_{0}
\end{aligned}
$$

From this Jensen-Peierls inequality $F \leq F_{v}$, we adjust the variational parameters contained in $E_{0}$ optimally so that $F_{v}$ is minimized.

For $E_{0}$ of the present system, we assume the translational invariance and consider the following sum of singlesite and single-link energies;

$$
E_{0}=-W \sum_{x} \sum_{\mu} J_{x \mu}-h \sum_{x} S_{x}
$$

where $W$ and $h$ are real variational parameters. Then we calculate the variational free energy per site, $f_{v} \equiv F_{v} / N$, where $N$ is the total number of lattice sites (we present the formulae for $d$-dimensional lattice) as

$$
\begin{aligned}
f_{v}= & -\frac{d}{\beta} \ln (2 \cosh \beta W)-\frac{1}{\beta} \ln (2 \cosh \beta h)-c_{1} d m^{2} M \\
& \quad-c_{2} \frac{d(d-1)}{2} M^{4}-4 c_{3} \frac{d(d-1)}{2} m^{2} M^{3}+d W M+h m \\
m \equiv & \left\langle S_{x}\right\rangle_{0}=\tanh \beta h \\
M \equiv & \left\langle V_{x \mu}\right\rangle_{0}=\tanh \beta W
\end{aligned}
$$

The stationary conditions $\partial f_{v} / \partial W=\partial f_{v} / \partial h=0 \mathrm{read}$

$$
\begin{aligned}
W & =c_{1} m^{2}+2 c_{2}(d-1) M^{3}+6 c_{3}(d-1) m^{2} M^{2}, \\
h & =2 d c_{1} m M+4 c_{3} d(d-1) m M^{3},
\end{aligned}
$$

which give rise to the equations for $m$ and $M$;

$$
\begin{aligned}
m= & \tanh \left[2 \beta d c_{1} m M+4 \beta c_{3} d(d-1) m M^{3}\right], \\
M= & \tanh \left[\beta c_{1} m^{2}+2 c_{2} \beta(d-1) M^{3}\right. \\
& \left.+6 \beta c_{3}(d-1) m^{2} M^{2}\right] .
\end{aligned}
$$

By assuming suitable scaling behavior of parameters $\beta c_{i}$ at large $d$, the result of MFT is known to become exact for $d \rightarrow \infty$ [25].

The MFT equations (4.4-4.6) generate the three phases characterized as follows;

\begin{tabular}{|c|c|c|c|c|}
\hline phase & $\left\langle J_{x \mu}\right\rangle_{0}$ & $\left\langle S_{x}\right\rangle_{0}$ & ability & Hopfield \\
\hline Higgs & $\neq 0$ & $\neq 0$ & learn and recall & ferromagnetic \\
\hline Coulomb & $\neq 0$ & 0 & learn & paramagnetic \\
\hline Confinement & 0 & 0 & N.A. & N.A. \\
\hline
\end{tabular}

In the first column of (4.7), the name of each phase is given, which is used in particle physics. The second and third columns show the order parameters of MFT, $\left\langle J_{x \mu}\right\rangle_{0}(=M),\left\langle S_{x}\right\rangle_{0}(=m)[26]$. The fourth column shows the properties of each phase. The condition $M \neq 0$ is a necessary condition so that a phase has the ability to learn a pattern $S_{x}=\xi_{i}^{\alpha}$ by storing it to $J_{x \mu}$. There the fluctuations of $J_{x \mu}$ should be small so that $J_{x \mu}$ generate a nontrivial minimum of $E$ at $S_{x}=\xi_{i}^{\alpha}$. ( $M=0$ means the fluctuations are too large.) The condition $m \neq 0$ is a necessary condition to recall the pattern $S_{x}=\xi_{i}^{\alpha}$. The fluctuations of $S_{x}$ around $S_{x}=\xi_{i}^{\alpha}$ should be small when recalling is successful. We note that the fourth phase with $M=0$ and $m \neq 0$ is missing. This sounds natural since learning should be a necessary condition for recalling, hence partly supporting the gauge symmetry of neural network. The fifth column indicates the corresponding phases in the Hopfield model.

In Fig. 6 we plot the phase diagrams obtained from (4.44.6 ) for various values of $c_{3}$. (The case of $c_{3}=0$ has been studied in Ref. [11].) The results of MC simulation in the 
next subsection are also presented by filled circles. The phase boundary of MFT between the Higgs phase and the Coulomb phase is second order, while other two boundaries, Higgs-confinement and confinement-Coulomb, are first order. In Fig.7 we present typical behaviors of $f_{v}$ for (a): second-order transition; (b) first-order transition. Across a second-order transition, $M$ and $m$ vary continuously, while across a first-order transition, $M$ and/or $m$ change discontinuously with finite jumps $\Delta M$ and/or $\Delta m$. For a Higgs-confinement transition, $\Delta M \neq 0$ and $\Delta m \neq 0$, and for a confinement-Coulomb transition, $\Delta M \neq 0$ and $\Delta m=0$ since $m=0$ in both phases.

The locations of phase boundaries of MFT agree globally with those of MC simulation in Sect.IVB. However, the results of MFT are not sufficient in the following two points;

(i) The MC simulation shows that the confinementCoulomb transitions are second-order contrary to the MFT. This point may be explained as $d=3$ is not large enough for MFT. The MC simulation for $d=4$ [27] for $c_{3}=0$ reports first-order confinement-Coulomb transitions as the MFT does.

(ii) The MC simulation shows that the Higgsconfinement boundary terminates at a certain end point. Along this phase boundary, the jumps $\Delta M, \Delta m$ decrease as $c_{2}$ decreases and disappear at the end point at $c_{2}>0$. This corresponds to the complementarity studied in Ref. [28] for $c_{3}=0$, which states that these two phases are analytically connected through a detour.

There are the following special cases that are examined better by other methods;

\section{CASE I: Ising model $\left(c_{2} \rightarrow \infty\right)$}

Here the $c_{2}$ terms forces $J_{x \mu}$ to the so-called pure-gauge configuration, and the energy reduces to that of the Ising model;

$$
\begin{aligned}
J_{x \mu} & \rightarrow V_{x+\mu} V_{x} \\
E & \rightarrow-\left(c_{1}+4 c_{3}\right) \sum_{x} \sum_{\mu} S_{x+\mu}^{\prime} S_{x}^{\prime}+\text { const. } \\
S_{x}^{\prime} & \equiv V_{x} S_{x}= \pm 1 .
\end{aligned}
$$

Thus, there is a second-order Ising transition at $\beta\left(c_{1}+\right.$ $\left.4 c_{3}\right) \simeq 0.22$ for $c_{2}=\infty$.

CASE II: Pure gauge model $\left(c_{1}=c_{3}=0\right)$

Here the energy is

$$
E=-c_{2} \sum_{x} \sum_{\mu>\nu} J_{x \mu} J_{x+\mu, \nu} J_{x+\nu, \mu} J_{x \nu}+\text { const. }
$$

This system is known [27] to exhibit a second-order transition at $\beta c_{2} \simeq 0.76$. Actually, after the duality transformation, the system (4.9) is converted to the 3D Ising spin model.
CASE III: Single-link model $\left(c_{2}=c_{3}=0\right)$

Here the sum over $J_{x \mu}$ is possible because the energy is decoupled to each link, and the partition function becomes an $S_{x}$-independent constant;

$$
\begin{aligned}
\sum_{J_{x \mu}= \pm 1} \exp \left(\beta c_{1} S_{x} J_{x \mu} S_{x+\mu}\right) & =2 \cosh \left(\beta c_{1} S_{x} S_{x+\mu}\right) \\
& =2 \cosh \left(\beta c_{1}\right)
\end{aligned}
$$

due to $S_{x}^{2}=1$. Thus the free energy has no singularities and no phase transitions along the $c_{1}$-line. This explains why the Higgs-confinement transition line should truncate at the end point (before reaching the $c_{1}$-line) as the complementarity [28] claims.

\section{CASE IV: Self-duality curve $\left(c_{3}=0\right)$}

For $c_{3}=0$, the standard $\mathrm{Z}(2)$ duality transformation can be applied [29], which maps the model at $P\left(\beta c_{1}, \beta c_{2}\right)$ onto the same model at $P^{\prime}\left(\beta c_{1}^{\prime}, \beta c_{2}^{\prime}\right)$ with

$$
\beta c_{1}^{\prime}=-\frac{1}{2} \operatorname{lnth}\left(\beta c_{2}\right), \beta c_{2}^{\prime}=-\frac{1}{2} \ln \operatorname{th}\left(\beta c_{1}\right) .
$$

If we assume the phase transition occurs at the point satisfying $P^{\prime}=P$, we obtain the curve of phase transition from (4.11) as

$$
\beta c_{2}=-\frac{1}{2} \operatorname{lnth}\left(\beta c_{1}\right) .
$$

In the next subsection, we shall see that a part of the curve (4.12) is actually the phase boundary.

\section{B. MC simulation}

We performed MC simulations for a 3D lattice of the size $N=L^{3}$ up to $L=16$ with the periodic boundary condition. The case of $c_{1}=c_{3}=0$ has been examined by Bahnot and Creutz. [27]. We employed MA, which is illustrated in Fig.5, with choosing the prefactors $\alpha_{S}=$ $\alpha_{J}=0.9$. Typical numbers of sweeps $[M$ of (3.7)] are $10^{5}$ for thermalization and $5 \times 10^{4}$ for measurements.

Among others, we measured the internal energy $U$ and the specific heat $C$,

$$
\begin{aligned}
& U=\langle E\rangle, \\
& C=\frac{d U}{d T}=\beta^{2}\left\langle(E-\langle E\rangle)^{2}\right\rangle .
\end{aligned}
$$

We judge the order of transition as follows; If $U$ has a discontinuity at the transition point, it is of first order. A typical behavior of $U$ and $C$ for a first-order transition is given in Fig.8a and Fig.8b. If $U$ is continuous and $C$ has a peak and discontinuity, then it is of second order. A typical behavior of $U$ and $C$ for a second-order transition is given in Fig.9a and Fig.9b. 
Let us comment on the gauge fixing. Our MC simulations have been done without fixing the gauge. We have also made MC simulations in the unitary gauge,

$$
S_{x}=1 .
$$

Although the partition function in (4.1) and the corresponding averages are independent whether one fixes the gauge or not, variations of the variables in Markov processes in the unitary gauge are too small and the convergence of the expectation values are too slow to obtain meaningful results with good accuracies. This slow convergence shall persist even for other gauge fixings.

\section{Column-layer structures in $c_{2}<0$}

Usual studies of lattice gauge theory have been restricted to the case of $c_{2}>0$. However, as a model of neural network, the case of $c_{2}<0$ is also interesting since this condition implies that the $c_{2}$-term in the energy expresses negative feed backs, that is, signals starting from $x$ will propagate around the plaquette and return to $x$ with a negative coefficient.

For $c_{2}<0$, the $c_{2}$-term prefers "flux states" of $J_{x \mu}$, i.e., $J_{x \nu} J_{x+\nu, \mu} J_{x+\mu, \nu} J_{x \mu}=-1$. Then it will generally compete with the $c_{1}$-term which prefers fluxless states, $J_{x \nu} J_{x+\nu, \mu} J_{x+\mu, \nu} J_{x \mu}=1$ [31]. Thus the system is frustrated. The MFT with the translationally-invariant variational energy of (4.3) is inadequate for this situation, and the MC simulation is necessary.

In Fig.10, we present the phase diagram of MC simulation for the extended region with $c_{2}<0$ and/or $c_{1}<0$. In the region $c_{2}<0$, there are phase boundaries that look like mirror images of $c_{2}>0$ of Fig. 6 except that the Higgs-confinement boundaries extend to $c_{1} \rightarrow \pm \infty$ instead of terminating at the ending critical points. The phase $\mathrm{F}$ in Fig.10 corresponds to the Higgs phase B, the phase $\mathrm{E}$ to Coulomb phase $\mathrm{A}$, and the region $\mathrm{D}$ is connected to the confinement phase C. For $c_{3}=0$ the phase diagram is symmetric w.r.t the $c_{1}=0$-line, reflecting the symmetry under $\left(c_{1}, c_{3}\right) \leftrightarrow\left(-c_{1},-c_{3}\right)$ [32], i.e.,

$$
Z\left(c_{1}, c_{2}, c_{3}\right)=Z\left(-c_{1}, c_{2},-c_{3}\right) .
$$

Bahnot and Creutz [30] performed MC simulations for $c_{2}<0$ at $c_{3}=0$ with the related interest of spinglass (though taking $J_{x \mu}$ as an annealed variable). Our result at $c_{3}=0$ is consistent with their result. At $c_{3} \neq 0$, the symmetry w.r.t. the $c_{1}=0$-line is violated as shown in Fig.10b for $\beta c_{3}=0.05$.

In the "Higgs" phase F, we observe a "column-layer" structure in the spatial configurations of the following link objects;

$$
j_{x \mu} \equiv\left\langle S_{x+\mu} J_{x \mu} S_{x}\right\rangle .
$$

$j_{x \mu}$ is the thermal average of a gauge invariant extension of $J_{x \mu}$ as appeared in the $c_{1}$-term of the energy. It measures the efficiency of signal propagations between $x$ and $x+\mu$. In Fig.11, we present the basic unit of a typical periodic configurations of $j_{x \mu}$ that forms a column-layer lattice in the phase $\mathrm{F}$.

This column-layer structure on the lattice is stable, because $j_{x \mu}$ are not snap shots but thermal (i.e., time) averages in long $\mathrm{MC}$ runs. It is to be spontaneously generated as a result of dynamics, i.e., a result of self-organization in the present model. In short, its origin is the frustration (competition) between the $c_{1}$ and $c_{2}$ terms in the energy.

There are several possible configurations of $j_{x \mu}$ which differ from that of Fig.11 in the direction of 1D alignments (i.e., other than in the 1-direction) and the orientation of the planes of layers (other than in the 1-2 plane). These options are generated by starting with different initial configurations (and/or different random numbers). In this sense, their generations are spontaneous, but they are stable once they are formed as we explained.

Since a large $j_{x \mu}$ means that signals (potential) propagate between $S_{x}$ and $S_{x+\mu}$ frequently and coherently, each column in the column-layer structure is a $1 \mathrm{D}$ path along which signals propagate dominantly. It is interesting to study possible relevance of such a structure to the actual self-organized structures observed in the human brains like ocular dominance columns. [5-8] (See the future problems in Sect.VI.) We also note that the present structure of weakly-coupled planes may be viewed as a kind of multilayer neural network.

\section{LEARNING AND ASSOCIATIVE MEMORY}

In the previous section, we studied the phase structure of the model, which reflects the static properties of the model. In this section, we simulate the processes of learning a pattern of $S_{x}$ and recalling it. The results reflect the dynamical properties of the model.

We set up the simulations in the following two steps in time;

(i) Learning during $0<t<t_{1}$ :

We first prepare a pattern $S_{x}=\xi_{x}$ to memorize and start with it, $S_{x}(0)=\xi_{x}$. During this learning time, we freeze $S_{x}(t)$ as

$$
S_{x}(t)=S_{x}(0) \text { for } 0<t<t_{1},
$$

by hand to let the system learn it. This may correspond to apply very strong stimuli to the brain like forcing it to watch the pattern with concentration. On the other hand, we allow $J_{x \mu}(t)$ vary according to MA to adjust themselves to a configuration suitable for $S_{x}(0)$. In terms of the prechoice parameters $\alpha_{S}, \alpha_{J}$ of MA (where $1-\alpha$ is a parameter to control the rate of time variation), we set $\alpha_{S}=1$ and $\alpha_{J}<1$ for $0<t<t_{1}$. Below we fix $\alpha_{J}=0.9$ 
for $0<t<t_{1}$. The time $t$ is defined throughout the simulations $(0<t)$ as $t \equiv\left(1-\alpha_{J}\left(0<t<t_{1}\right)\right) \times$ number of iterations (sweeps), i.e., $t \equiv 0.1 \times$ number of iterations.

(ii) Recalling during $t_{1}<t$ :

At $t=t_{1}$ we change $S_{x}$ discontinuously from $S_{x}(0)$ to a pattern $S_{x}\left(t_{1}\right)$ that is obtained by adding a certain amount of random noise upon $S_{x}(0)$. For definiteness, we set $S_{x}\left(t_{1}\right)=-S_{x}(0)$ for the $10 \%$ of sites $x$ that are randomly chosen throughout the entire lattice (except for Fig.17 discussed later where we consider the cases of more amounts of noises). This forced change of $S_{x}(t)$ at $t=t_{1}$ simulates relaxing the brain from the concentration upon $S_{x}(0)$ and letting it forget $S_{x}(0)$ partly. Then we let $S_{x}(t)$ vary according to MA with $\alpha_{S}<1$. Below we fix $\alpha_{S}=0.9$ for $t_{1}<t$. On the other hand, $J_{x \mu}(t)$ basically vary according to MA with $\alpha_{J}<1$ smoothly starting from $J_{x \mu}\left(t_{1}\right)$. However, with the reason explained below, we shall be also interested in the special limit $\alpha_{J} \rightarrow 1$ in which the time variations of $J_{x \mu}(t)$ after $t_{1}$ freeze;

$$
\alpha_{J} \rightarrow 1: J_{x \mu}(t)=J_{x \mu}\left(t_{1}\right) \text { for } t_{1}<t .
$$

To judge the performance of each process of learning a pattern and recalling it in a quantitative manner, we use the following overlaps;

$$
\begin{aligned}
O_{S}(t) & \equiv \frac{1}{N} \sum_{x} S_{x}(0) S_{x}(t), \\
O_{J}(t) & \equiv \frac{1}{3 N} \sum_{x} \sum_{\mu} J_{x \mu}(0) J_{x \mu}(t),
\end{aligned}
$$

which are gauge-invariant under (3.3). If the recalling is successful, we expect $S_{x}(t) \simeq S_{x}(0)$ at sufficiently large $t$, so $O_{S} \simeq 1$. (Note that $O_{S}\left(t_{1}\right)=0.8$ for the $10 \%$ change of $S_{x}$ at $t=t_{1}$.) On the other hand, $O_{J}\left(t_{1}\right)$ measures the rate how much the synaptic connections change by learning during $0<t<t_{1}$. In Fig.12, we illustrate $O_{S}$ and $O_{J}$ versus $t$ in two typical processes for the case (5.2). Fig.12a is a process that succeeds in recalling, and Fig. $12 \mathrm{~b}$ is one that fails in recalling.

Let us first study the dependence of the overlaps upon the choice of $\alpha_{J}$ for $t_{1}<t$. In Fig.13 we plot $O_{S}(t)$ and $O_{J}(t)$ for three cases, $\alpha_{J}=1.0,0.9,0.99$ for $t_{1}<t$. (Note that we fixed $\alpha_{J}=0.9$ for $0<t<t_{1}$.) As the initial condition, we choose $S_{x}(0)$ and $J_{x \mu}(0)$ randomly. We select three points in the parameter space of $c_{i}$. Fig.13a is the result for $c_{1}=1, c_{2}=c_{3}=0$ which belongs to the confinement phase. For $\alpha_{J}=1\left(J_{x \mu}(t)=J_{x \mu}\left(t_{1}\right)\right), O_{S}$ approaches to the constants near 0.95 ; the system (almost) succeeds to recall $S_{x}(0)$. For $\alpha_{J}=0.99, O_{S}$ once increases and then decreases. It describe a process of recalling $S_{x}(0)$ partly and then lose the memory gradually, that is, the phenomenon of a partial memory loss(deficit). For $\alpha_{J}=0.9, O_{S}$ decreases monotonically. These latter two curves exhibit a typical phenomenon of a dynamical system with coupled variables. A fixed point obtained by fixing some variables may become unstable when all are coupled. As expected, $O_{S}$ decreases more as $J_{x \mu}$ change more rapidly (i.e., $\alpha_{J}$ decreases more). Fig.13b is for the point $c_{1}=3.0, c_{2}=1.0, c_{3}=0$, and Fig. $13 \mathrm{c}$ is for the point $c_{1}=3.0, c_{2}=-1.0, c_{3}=0$, both of which are in the Higgs phase. In contrast to Fig.13a, $O_{S}$ in these points have smaller dependence on $\alpha_{J}$, so the effects of memory loss are smaller than in the confinement phase. Although the time-variations of $J_{x \mu}(t)$ for $t_{1}<t\left(\alpha_{J} \neq 1\right)$ reduce the performance of recalling more (in the deconfinement phase) or less (in the Higgs phase), we stress that these phenomena of partial memory loss are not a flaw of the present model, but a welcome feature that the realistic model of human brain should possess.

Since the case of $\alpha_{J}=1$ for $t_{1}<t$ gives the stable and highest values of $O_{S}$ at large $t$, we present the results for $\alpha_{J}\left(t_{1}<t\right)=1$ in Fig.14-18 [33] below as typical results for $\alpha_{J} \simeq 1$. This choice $\alpha_{J}\left(t_{1}<t\right)=1$ for presentations sounds also reasonable because the actual variations of synaptic connections, $J_{x \mu}(t)$, in the human brain are much slower than those of $S_{x}(t)$ as explained in Sect.IIIB, i.e., $\alpha_{J} \simeq 1$. (In this viewpoint, our choice $\alpha_{J}=0.9$ for $0<t<t_{1}$ corresponds to accelerate the learning processes significantly. If we take larger $\alpha_{J} \simeq 1$ for $0<t<t_{1}$, then we need to take $t_{1}$ larger in order to obtain the same values of $O_{S}$.)

Next we study the dependence on the initial conditions. In Fig. 14 we present the contours of $O_{S}$ at large $t$ for four choices (a-d) of the initial states $S_{x}(0), J_{x \mu}(0)$. The results of first three choices (a-c) look similar each other, while the fourth case (d) $S_{x}(0)=1, J_{x \mu}(0)=1$ is different; the region of higher $O_{S}$ for $c_{2}>0$ extends to the phase boundary $\left(c_{1} \simeq 0.4\right)$ of the Higgs phase and the Coulomb phase. This is natural since the configuration $S_{x}=J_{x \mu}=1$ is the minimum of the free energy in the Higgs phase at $c_{2}>0$. Thus the system has learned this pattern $S_{x}(0)=1$ from the beginning. Below we present the result for the random initial condition, the choice (a), i.e., $S_{x}(0)$ and $J_{x \mu}(0)$ are chosen randomly from \pm 1 .

To study the dependence on the learning time $t_{1}$, we present in Fig.15 two typical processes with different $t_{1}$. In Fig. $15 \mathrm{a}, t_{1}=1$ and $O_{S}$ approaches to $O_{S}=0.6$, so it fails to recall $S_{x}(0)$. On the other hand, in Fig.15b, $t_{1}=5$ and $O_{S}$ approaches to $O_{S}=0.92$, so we judge it succeeds (but not completely) to recall $S_{x}(0)$. A reason is clearly drawn from the behavior of $O_{J}$. In Fig.15b, $O_{J}$ almost converges to a fixed value at $t_{1}$, that is $J_{x \mu}$ converge to the suitable configuration, while, in Fig.15a, $O_{J}$ is in a way to converge. Thus, a necessary condition to learn successfully is to keep $t_{1}$ sufficiently long (longer than the relaxation time of $J_{x \mu}$ ) so that $J_{x \mu}$ can converge to the configuration that makes $S_{x}=S_{x}(0)$ a minimum of the energy. For definiteness we present the results for $t_{1}=5$ below.

Let us next study the relative importance of each term $c_{1}, c_{2}, c_{3}$ of the energy in learning and recalling. In Fig.16, $O_{S}$ after sufficiently large time is plotted for three cases where only one $c_{i}$ of $c_{1}, c_{2}, c_{3}$ is nonvanishing and the 
other two are zero. In the case of $c_{1}$ alone, as $c_{1}$ increases, $O_{S}$ approaches unity. This is expected since the $c_{1}$-term describes the direct transfers of signals. In the case of $c_{3}$ alone, as $c_{3}$ increases, $O_{S}$ increases but saturates around $O_{S} \simeq 0.65$. This indicates that the indirect signal transfers by the $c_{3}$-term is not sufficient by itself to recall the original pattern, as anticipated. In the case of $c_{2}$ alone, $O_{S} \approx 0$. This is natural because the $c_{2}$-term, which contains only $J_{x \mu}$ and no $S_{x}$, expresses signal-transfers starting from a neuron and ending at the same neuron through a loop along a plaquette, but describes no signal-transfers to the NN neurons.

Let us see the roles of $c_{2}, c_{3}$-terms in details. Since we have observed that the $c_{1}$-term plays the central role in learning and recalling, we simulate the processes with $c_{1}+c_{2}$ and $c_{1}+c_{3}$. In Fig. 17 we plot $O_{S}$ after large time for several $S_{x}\left(t_{1}\right)$ 's prepared by adding certain amounts of noises to $S_{x}(0)$ from $10 \%$ up to $40 \%$. It shows that adding certain amounts of $c_{2}$ or $c_{3}$ upon $c_{1}$ improves the rate of recalling, i.e., to achieve larger $O_{S}$. It is interesting to note that there is a preferred region for $c_{2}$, $\beta c_{2} \in(0.2,0.6)$, for which $O_{S}$ is over $95 \%$. This reminds us the phenomenon that applying a certain but not too much amount of magnetic field improves our brain activities.

Let us study the relation between the results of present section and the phase structure of the previous section. In Fig.18, we superpose contour plots of $O_{S}$ on the phase diagram Fig.6b. We observe that being in the Higgs phase is not sufficient to achieve good rates of successful learning and recalling. This is clear especially in the region with large $c_{2}$, which is consistent with the case of $c_{2}$ alone in Fig.16. Also, in the region near $\beta c_{1}=0.5, \beta c_{2}=0.4$ which is a vague border between the confinement and the Higgs phases, learning is possible. So this region may have something to do with our experience that a tiny amount of stimulation helps us to recall certain patterns; a coexisting phenomenon of recalling and nonrecalling. Apart from these regions, there are certainly the correlations among the results of this section and the phase diagram of Sect.III.

From these results, one may list up the conditions to succeed in learning a pattern of $S_{x}$ and recalling it as follows;

- The learning time $t_{1}$ should be larger than the relaxation time of $J_{x \mu}$.

- The temperature $T$ should be low.

- The self-interaction term $\beta c_{2}$ should be within a certain range $\left(0.0<\beta c_{2}<0.7\right.$ for $\left.\beta c_{1}=1.0\right)$.

- The indirect $c_{3}$-term should be of the same signature as the direct $c_{1}$-term to accelerate signal transfers caused by the $c_{1}$-term.

\section{CONCLUSIONS AND FUTURE PROBLEMS}

By converting the strength $J_{i j}$ of the synaptic connection to a $\mathrm{Z}(2)$ gauge variable (exponentiated gauge connection) and imposing the gauge symmetry on the energy $E\left(\left\{S_{i}\right\},\left\{J_{i j}\right\}\right)$, we have proposed an explicit model of neural network of learning. The Z(2) gauge symmetry is inherited from the Hopfield model. Study of the phase structure and the simulations of learning and recalling revealed several interesting features of the model.

- In the confinement phase, both learning and recalling are disabled, which may corresponds to certain symptoms like Alzheimer's disease [See (4.7)]. The complementarity characterized by the end point of Higgs-confinement phase-transition curve may offer us some methods to retrieve the network from the confinement phase to the Higgs phase in a continuous and practical manner.

- The column-layer structure of $\left\langle S_{x+\mu} J_{x \mu} S_{x}\right\rangle$ discussed in Sect.IVC implies that there are particular 1D paths (columns) in each layer along which the signals (electric potential) propagate dominantly. This structure seems to exhibit the potentiality of the present model to evolve the self-organized column structures of the active neurons, which are observed in the human brains [5-8].

- Due to the mutual interactions between $S_{x}$ and $J_{x \mu}$, the phenomena of memory loss are observed as in Fig.13. - The time evolution of $J_{x \mu}$ generalizes the Hebbian rule of learning. Suitable amounts of the newly added $c_{2}, c_{3^{-}}$ terms improve the performance of learning and recalling (Fig.17).

We think that these features make the present model interesting as a neural network of learning and associative memory and worth to make further investigations.

There are certainly various ways to improve the present model. Among others, we list up the following;

(1) Actual synaptic connections are not symmetric, i.e., $J_{i j} \neq J_{j i}$. However, in the Hopfield model, due to the very form of its energy $E$ of (1.2), the antisymmetric part of $J_{i j}, J_{i j}-J_{j i}$, does not contribute to $E$. This flaw can be removed in the present framework by introducing two independent $Z$ (2) variables, $J_{x \mu}$ and $J_{x+\mu,-\mu}$, on a link $(x, x+\mu)$. Then the $c_{2}$ and $c_{3}$ terms can reflect the antisymmetric part $J_{x \mu}-J_{x+\mu,-\mu}$. In fact, an asymmetric model in this direction is proposed in Ref. [11] (Model III) and its phase diagram is studied in MFT.

(2) In the present model, synaptic connections are restricted only to the NN neurons. In human brain, each neuron receives signals from $1000 \sim 10000$ neurons. These long-range connections are certainly responsible to store many patterns and should be incorporated in a more realistic model. We plan to increase the number of connections in the future study. This means to introduce more variables $J_{i j}$ beyond NN pairs. Related to this point, one may increase the number of patterns to memorize as $\xi_{x}^{\alpha}$ in the Hopfield model.

(3) Natural ways to incorporate external stimuli like visual and acoustic ones to the present model may be (i) 
to change the boundary condition from the present periodic boundary condition to a fixed boundary condition with an appropriate values of $S_{x}, J_{x \mu}$ on the surface of the 3D lattice, and/or (ii) fixing $S_{x}, J_{x \mu}$ in certain part of the system to certain constants. By studying the response to each stimulation, one may address the question whether column structures of active neurons are generated in the present model.

\section{Acknowledgments}

We thank Yukari Fujita and Yuki Nakano for discussion. One of the authors (K.S.) thanks the members of Department of Physics, Kanazawa University for their hospitality delivered to him during his stay.

\section{Note added}

The gauge model of neural network has been extended to a quantum neural network having U(1) gauge symmetry in Y.Fujita and T.Matsui, Proceedings of 9th International Conference on Neural Information Processing, ed. by L.Wang et al. (2002)1360-1367 (arXiv:condmat/0207023).

[1] J.J.Hopfield, Proc.Nat.Acd.Sci.USA.79,2554(1982).

[2] D.O.Hebb, "The Organization of Behavior: A Neuropsychological Theory", New York: Wiley. (1949)

[3] Here $\xi_{i}^{\alpha}$ 's are assumed to be orthogonal each other, i.e., $N^{-1} \sum_{i} \xi_{i}^{\alpha} \xi_{i}^{\beta}=\delta_{\alpha \beta}$. For $M>0.14 N$ there appear unwelcome energy minima other than $S_{i}=\xi_{i}^{\alpha}$.

[4] F.Rosenblatt, "Principles of Neurodynamics-Perceptrons and the Theory of Brain Mechanisms", Spartan, 1961.

[5] J.A.Anderson, Math.Bio-Sci., 8,137(1970); ibid.14,197(1972); S.Amari and A.Takeuchi, Biological Cybernetics,29,127(1978); K.D.Miller,J.B.Keller and M.P.Stryker, Science,245,605(1989).

[6] C.von der Malsburg, Kybernetik, 14,85(1974);

[7] S.Tanaka, Neural Networks 3, 625(1990); S.Tanaka and H.Shinbata, Biological Cybernetics 70, 227(1994).

[8] R.Linsker, Proc.Natl.Acad.Sci.USA, 83,7508(1986), ibid. 8390,8779 .

[9] For Boltzmann machine, Boltzmann learning rule and other models of learning, see S.Haykin, "Neural Networks; A Comprehensive Foundation", Macmillan Pub.Co.(1994).

[10] See, e.g., T.Ikegami and M.Suzuki, Prog. Theor. Phys.78, 38(1987).

[11] T.Matsui, pp. 271 in Fluctuating Paths and Fields, ed. by W.Janke et al., World Scientific (2001) (condmat/0112463).

[12] K.Wilson, Phys.Rev. D10, 2445(1974).

[13] J.B.Kogut, Rev.Mod.Phys. 51, 659(1979).
[14] A.Yu.Kitaev, in Proceedings of the Third International Conference on Quantum Communication and Measurement, ed. O.Hirota et al. (New York, Plenum, 1997); A.Yu.Kitaev, Ann.Phys. 303, 2(2003); M.Freedman, A.Kitaev, M.Larsen, and Z.Wang, quant-ph/0101025; J.Preskill, quant-ph/9712048; E.Dennis, A.Kitaev, A.Landahl, and J.Preskill, J.Math.Phys.43, 4452 (2002); C.Wang, J.Harrington and J.Preskill, Ann.Phys.303, 31 (2003); T.Ohno, G.Arakawa, I.Ichinose, and T.Matsui, Nucl.Phys.B697, 462 (2004).

[15] In Refs. [14], the ordered phase of $Z(2)$ gauge dynamics is called the Higgs phase since the mass of gauge field is massive there. This phase corresponds to Coulomb phase in the present model, because $\left\langle S_{i}\right\rangle=0$. The name Higgs phase is reserved for the phase with $\left\langle S_{i}\right\rangle \neq 0$ [see (4.7)].

[16] G.Toulouse, Commun. Phys.2,115(1977).

[17] S.F.Edward and P.W.Anderson, J.Phys.F5, 965(1975); D. Sherrington and S. Kirkpatrick, Phys. Rev. Lett. 35, 1792 (1975); M.Mezard, G.Parisi and M.A.Virasoro, "Spin Glass Theory and Beyond", World Scientific, Singapore (1987).

[18] See, e.g., J.Vannimenus and G.Toulouse, J.Phys. C10, L537 (1977); L.G.Marland and D.D.Betts, Phys. Rev. Lett.43, 1618 (1979); H.Nishimori and P.Sollich, J.Phys.Soc. Jpn. 69, A160 (2000); A.Keren and J.S.Gardner, Phys.Rev.Lett. 87,177201 (2001).

[19] More faithful generalization of the Tanaka model [7] may lead us to the variable $J_{i j}=-1,0,1$. (The constraint $\sum_{j} J_{i j}=1$ is to be irrelevant since the signature of $J_{i j}$ is indefinite here.) Such a case is discussed in [11] as the Model II on a 3D lattice. We made also some MC simulations for this case with the energy given in Sect.IIIA, which predict phase diagrams and a column structure similar to the case of $\mathrm{Z}(2)$ gauge variables presented in Sect.IVB and IVC.

[20] These considerations parallels the $\mathrm{SU}(2)$ isospin symmetry of a nucleon which has two states, proton and neutron, and associated Yang-Mills gauge theory. See W.Heisenberg, Z.Physik 77,1(1932); E.Wigner, Phys.Rev. 51,106(1837); B.Cassen and E.U.Condon, Phys.Rev. 50,846(1936); C.N.Yang and R.L.Mills, Phys.Rev.96,191(1954).

[21] N.G.van Kampen, "Stochastic Processes in Physics and Chemistry", North-Holland (1981).

[22] N.Metropolis, A.W.Rosenbluth, M.N.Rosenbluth, A.M.Teller, E.Teller, J.Chem.Phys. 21,1087(1953).

[23] Although $S_{x}$ and $J_{x \mu}$ are not continuous but discrete variables, $\Delta E$ is proportionalto the formal derivative like $\partial E / \partial S_{x}$.

[24] R. P. Feynman, "Statistical Mechanics, A set of Lectures", Chap.8, W. A. Benjamin (1972).

[25] J.M.Drouffe, Nucl.Phys.B170, 211 (1980).

[26] Elitzur [S.Elitzur, Phys.Rev.D12, 3978 (1975)] showed that expectation values of gauge-variant objects should vanish, thus $\left\langle S_{x}\right\rangle=\left\langle J_{x \mu}\right\rangle=0$. To compromise the MFT results with this Elitzur's theorem, Drouffe [25] proposed just to average over the gauge-transformed copies of a MF solution (4.6). Actually, the gaugetransformed variational energy, $E_{0}^{\prime}=-W \sum_{x} \sum_{\mu} J_{x \mu}^{\prime}-$ $h \sum_{x} S_{x}^{\prime}$, has the degenerate free energy $f_{v}^{\prime}(m, M)=$ 
$f_{v}(m, M)$. Thus it is allowed to take the average $\left\langle J_{x \mu}\right\rangle_{0} \equiv$ $2^{-N} \prod_{y} \sum_{V_{y}} V_{x+\mu} m V_{x}=0$, etc., which satisfies the Elitzur's theorem. The thermodynamic quantities, hence the location and the nature of phase transitions, are unchanged by this averaging.

[27] G.Bahnot and M.Creutz, Phys.Rev. D21, 2892 (1980). Creutz has also examined the case $c_{3}=0$ on a $4 \mathrm{D}$ lattice; M.Creutz, Phys.Rev. D21, 1006 (1980).

[28] E.Fradkin and S.Shenker, Phys.Rev.D19,3682 (1979).

[29] F.Wegner, J.Math.Phys.12, 2259 (1971).

[30] G.Bhanot and M.Creutz, Phys. Rev. B22, 3370 (1980).

[31] A simple way to see this is to make an expansion in powers of $c_{1}$ (assuming it is small). After the $S_{x}$ summation, a plaquette term like the $c_{2}$ term is generated at $O\left(c_{1}^{4}\right)$ with a coefficient favoring the fluxless states.

[32] The sites of 3D lattice are partitioned into odd sites and even sites according to the parity $(-)^{x_{1}+x_{2}+x_{3}}$. By making changes of variables $S_{x} \rightarrow S_{x}^{\prime} \equiv(-)^{x_{1}+x_{2}+x_{3}} S_{x}$ the energy changes the signatures of the $c_{1}$ and $c_{3}$ terms. $Z$ must be invariant under this changes of variables, which proves (4.15).

[33] We also drew some figures using $O_{S}$ at a suitable large time which are calculated with $\alpha_{J}\left(t_{1}<t\right)<1$. As expected from Fig.13, they deviate systematically from Fig.14-18. However, they look qualitatively similar each other, and we found no significantly new phenomena.

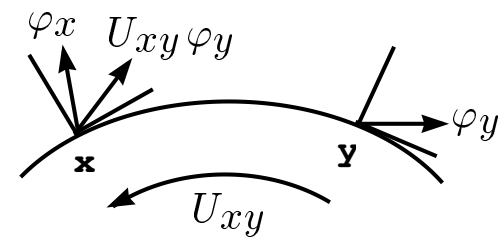

FIG. 1. Function of gauge variable $U_{x y}$ (path-dependent phase factor). $U_{x y}$ parallel-translates a vector $\varphi_{y}$ at the point $y$ to another point $x$ giving rise to $U_{x y} \varphi_{y}$. To compare two vectors $\varphi_{x}$ and $\varphi_{y}$, one needs to refer to the common local frame, say the frame at $x$. So one should parallel-translate $\varphi_{y}$ to $x$ and take the gauge invariant scalar product $\left(\varphi_{x}, U_{x y} \varphi_{y}\right) \equiv \varphi_{x}^{\dagger} U_{x y} \varphi_{y}$ instead of $\left(\varphi_{x}, \varphi_{y}\right)=\varphi_{x}^{\dagger} \varphi_{y}$.

(a)

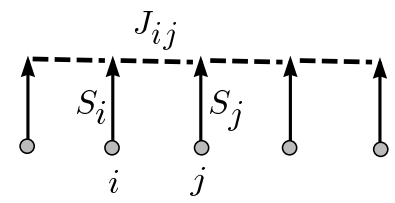

(b)

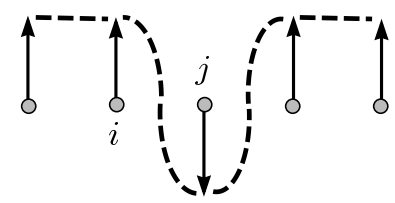

FIG. 2. Illustration of the local frames of $S_{i}$. Each solid line with an arrow indicates $S_{i}(= \pm 1$ ) at the site $i$ (filled circle). Each dashed line indicates the gauge variable $J_{i j}$ which measures the relative orientation of local frames at nearest-neighbor points. (a) Localframes where all the frames are in the same orientation. (b) Local frames after the local gauge transformation at $j, S_{j}^{\prime}=-S_{j}$. The information of this change is stored(absorbed) in the neighboring gauge variables $J_{i j}$ so that the signal received at $i$ is unchanged $J_{i j}^{\prime} S_{j}^{\prime}=J_{i j} S_{j}$.
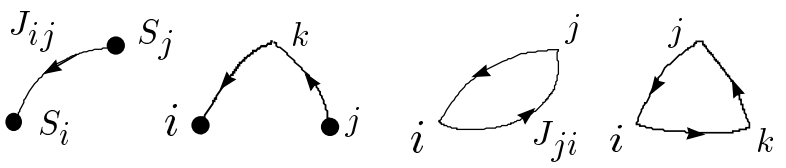

FIG. 3. Examples of gauge-invariant terms. The black circles denote $S_{i}$ and the curves with arrows denote $J_{i j}$. The figures indicate $S_{i} J_{i j} S_{j}, S_{i} J_{i k} J_{k j} S_{j}, J_{i j} J_{j i}, J_{i j} J_{j k} J_{k i}$ from the left, respectively. The first $S J S$ term is just the term of the Hopfield model. The second SJJS term may be viewed to describe the two successive processes $S_{k} J_{k j} S_{j}$ and $S_{i} J_{i k} S_{k}$. The last two terms consist of $J$ 's only, and describe closed circuits, which may be taken as "reverberating circuits". 


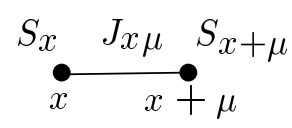

(a)

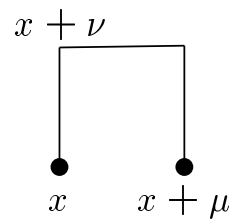

(c)

FIG. 4. Graphical representation of each term in $E$ of (3.4). The black circles represent $S_{x}$ and the line segments represent $J_{x \mu}$. Fig.a describes the direct interaction between $S_{x}$ and $S_{x+\mu}$ through $J_{x \mu}$. Fig.b is taken as the smallest "reverberating circuit". Fig.c describes the combined effects of three successive processes given by Fig.a.

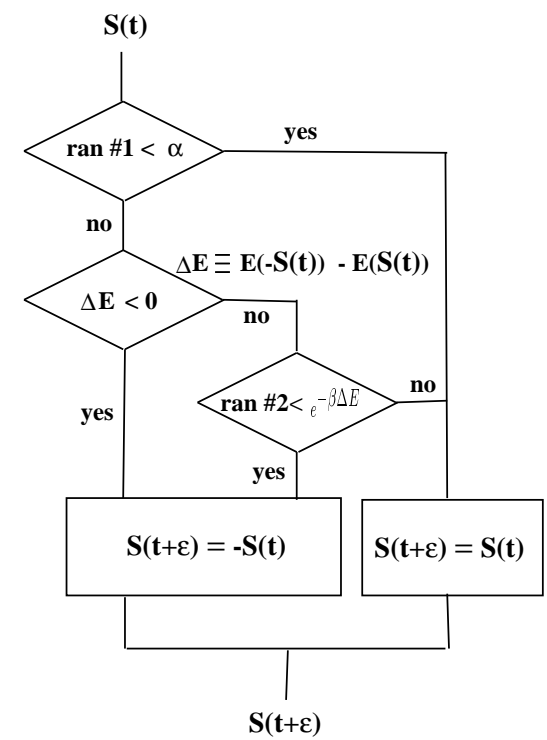

FIG. 5. Flow chart of Metropolis algorithm for a system with $\mathrm{Z}(2)$ variables $S= \pm 1$ to determine the value $S(t+\epsilon)$ starting from $S(t)$. ran\#1, ran\#2 are random numbers distributed uniformly in the interval $(0,1)$. This update process is to be done for each variable for every time step. In the present model, each update at every time step sweeps out all $S_{x}(t), J_{x \mu}(t)$ throughout the entire lattice.

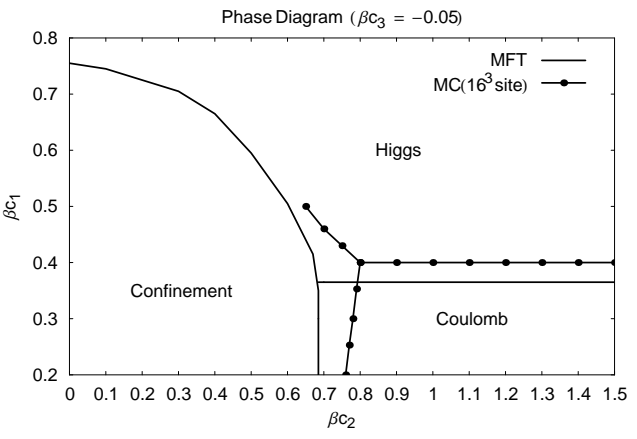

(a)

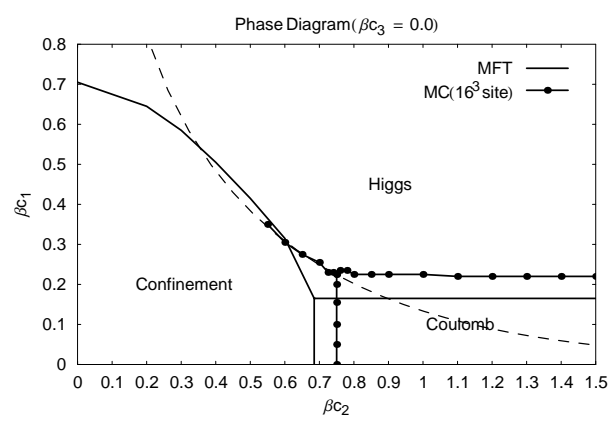

(b)

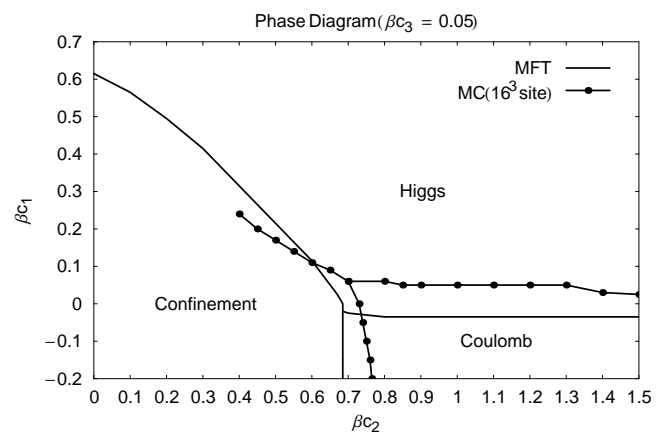

(c)

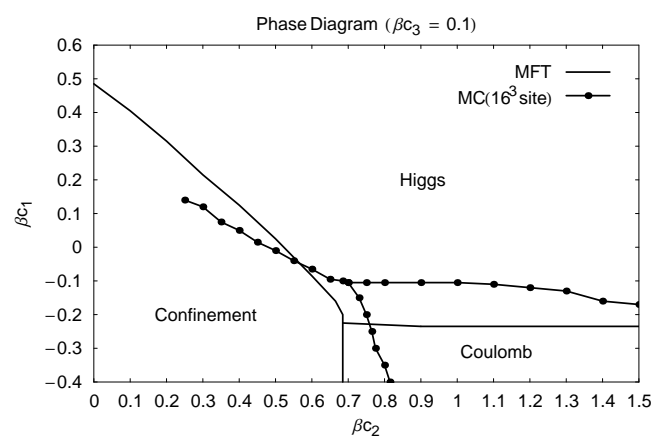

(d) 
FIG. 6. Phase diagram by MFT and MC simulation. (a) $\beta c_{3}=-0.05$, (b) $\beta c_{3}=0.0$, (c) $\beta c_{3}=0.05$, (d) $\beta c_{3}=0.1$. MC simulations show that the Higgs-Coulomb transition and the confinement-Coulomb transition are second order, while the Higgs-confinement transition is first order. The MFT predicts the first-order confinement-Coulomb transition incorrectly. Also the Higgs-confinement boundaries terminate at certain critical points instead of extending to $c_{2}=0$. The dashed curve in (b) is the phase-transition curve (4.12) predicted by the duality transformation. It almost agrees with our MC result in the period $0.55<\beta c_{2}<0.75$, in which the assumption $P^{\prime}=P$ for phase transitions is verified.

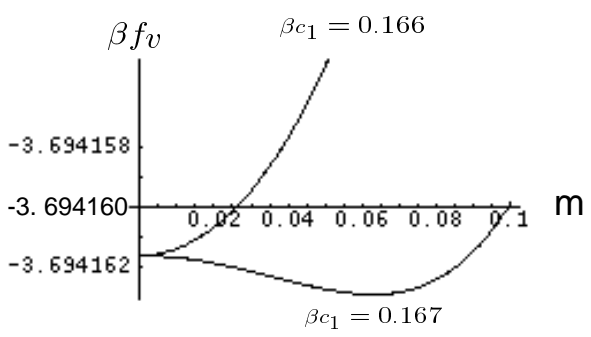

(a)

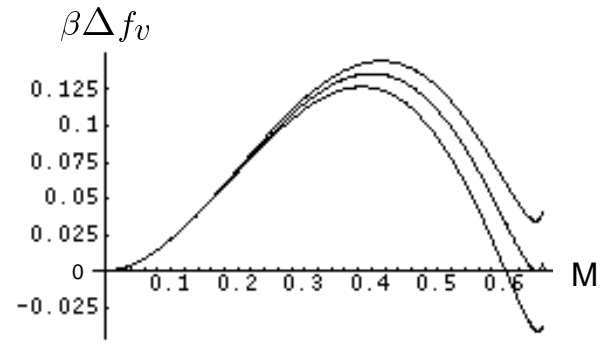

(b)

FIG. 7. Typical behavior of free energy $f_{v}$ of (4.4) near phase transitions. (a) $\beta f v(m, M)$ versus $m=\left\langle S_{x}\right\rangle$ for $\beta c_{1}=1, c_{3}=0$ near the second-order transition at $\beta c_{2}=0.166$. We set $M=\left\langle J_{x \mu}\right\rangle$ at the value on the transition point, $M=0.999$. (b) $\beta \Delta f_{v} \equiv \beta\left(f_{v}(m, M)-f_{v}(0,0)\right)$ versus $M$ for $\beta c_{2}=0.1, c_{3}=0$ near the first-order transition at which $\beta c_{1}=0.678, m=m_{c}=0.989, M=M_{c}=0.648$. The curves are drawn along the line $m=\left(m_{c} / M_{c}\right) M$ in the $(m, M)$ plane. The three curves are for $\beta c_{1}=0.66,0.678,0.70$ from above.

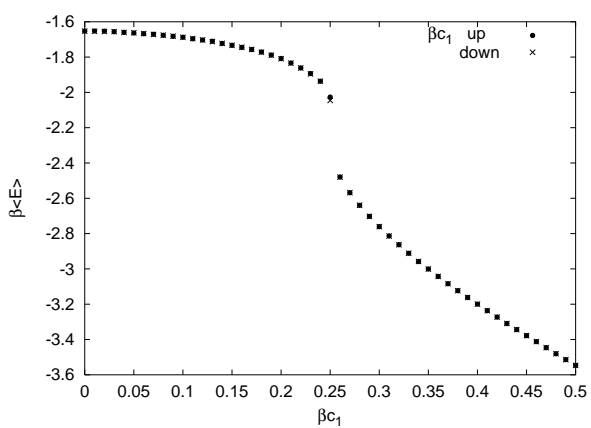

(a) $\beta\langle E\rangle / \mathrm{N}$ vs $\beta c_{1}$.

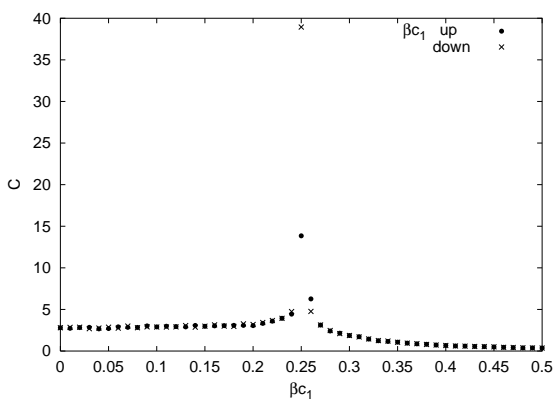

(b) $C / \mathrm{N}$ vs $\beta c_{1}$.

FIG. 8. Internal energy and specific heat per site versus $\beta c_{1}$ for a typical first-order transition $\left(c_{3}=0.0, \beta c_{2}=0.7\right)$. In the measurement, $\beta c_{1}$ is increased first (filled circles) and then decreased (crosses).

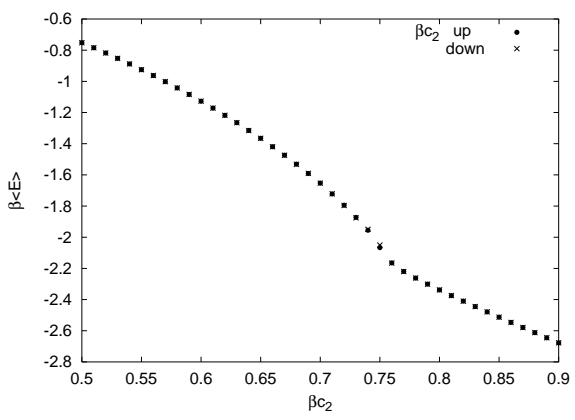

(a) $\beta\langle E\rangle / N$ vs $\beta c_{2}$.

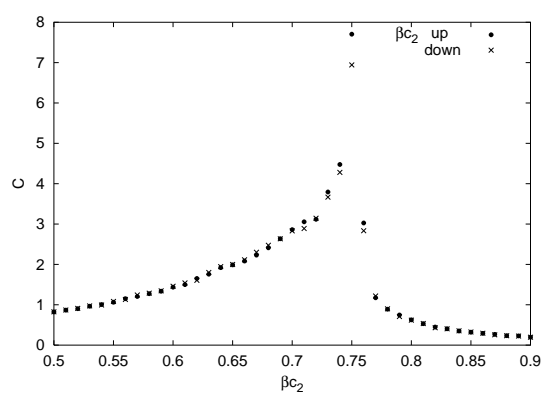

(b) $C / N$ vs $\beta c_{2}$.

FIG. 9. Internal energy and specific heat per site versus $\beta c_{2}$ for a typical second-order transition $\left(\beta c_{1}=0.1, c_{3}=0.0\right)$. In the measurement, $\beta c_{2}$ is increased first (filled circles) and then decreased (crosses). 


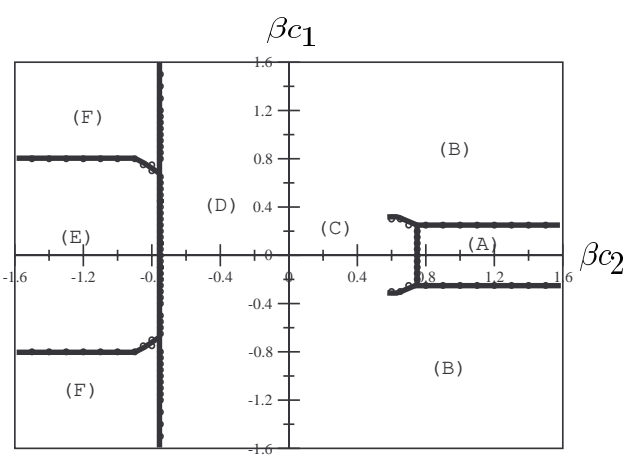

(a) $c_{3}=0$

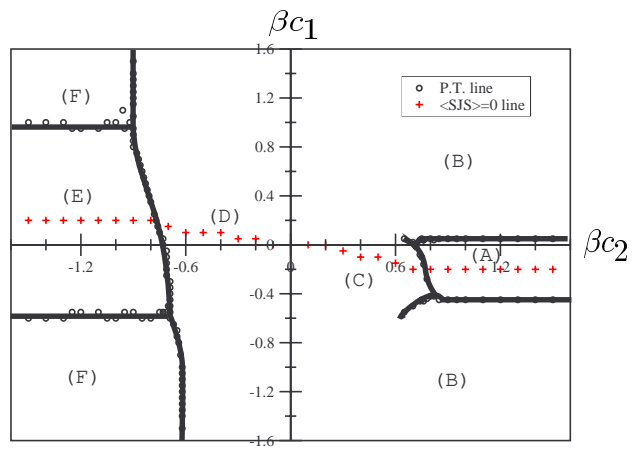

(b) $\beta c_{3}=0.05$

FIG. 10. Phase diagram including the extended regions $c_{1}, c_{2}<0$ for (a) $c_{3}=0$ and (b) $\beta c_{3}=0.05$. The phases $\mathrm{D}, \mathrm{E}, \mathrm{F}$ in $c_{2}<0$ are "mirror images" of C,B,A in $c_{2}>0$, respectively. Crosses in (b) indicate the points at which $j_{x \mu}=0$. (For the case $(\mathrm{a}), j_{x \mu}=0$ along the line $\beta c_{1}=0$.)

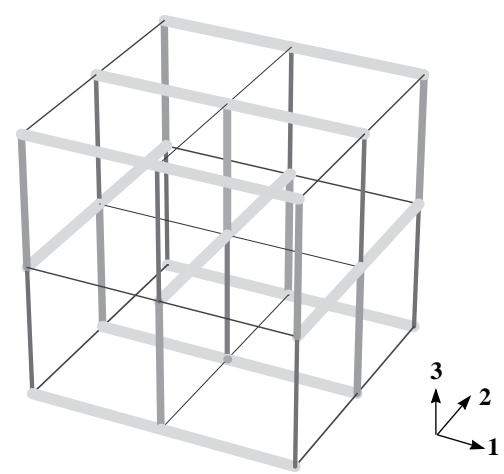

FIG. 11. Basic unit of the column-layer structure in the "Higgs" phase $\mathrm{F}$ in $c_{2}<0$. The entire configuration of $j_{x \mu} \equiv\left\langle S_{x+\mu} J_{x \mu} S_{x}\right\rangle$ is just the repetition of this $2 \times 2 \times 2$ structure in every direction. The thickness of each segment represents the magnitude of $j_{x \mu}$. Each plane with a certain orientation (the 1-2 plane here)has a one-dimensional columnic alignment of links with large (thick) $j_{x \mu}$ (say, in the 1-direction in the upper plane). In the next (middle) plane, the direction of the columns with large $j_{x \mu}$ is rotated by 90 degrees (in the 2-direction), and so on. Each pair of successive planes are weakly coupled by small (thin) $j_{x \mu}$ (in the 3 -direction).

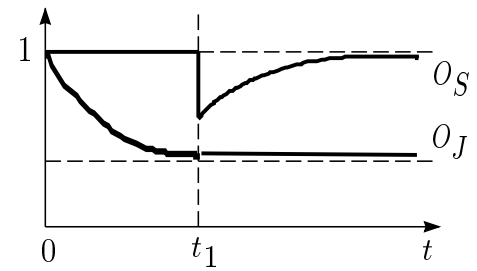

(a)

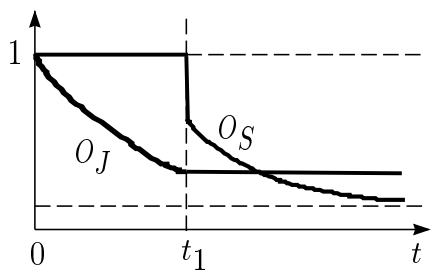

(b)

FIG. 12. Simulation of learning and recalling. For $0<t<t_{1}, S_{x}(t)$ is fixed to $S_{x}(0)$, and the system learns the pattern $S_{x}(0)$ by changing $J_{x \mu}(t)$. At $t=t_{1}$, we disturb $S_{x}$ to $S_{x}\left(t_{1}\right)$ discontinuously, where $S_{x}\left(t_{1}\right)$ is obtained by adding a certain amount of random noise to $S_{x}(0)$. For $t_{1}<t$, the system tries to recall $S_{x}(0)$ by changing $S_{x}(t)$. (We illustrate the case that $J_{x \mu}(t)$ for $t_{1}<t$ is fixed to $J_{x \mu}\left(t_{1}\right)$.) (a) It succeeds to recall $S_{x}(0)$ with $S_{x}(t) \simeq S_{x}(0)$ and $O_{S} \simeq 1$. (b) It fails to recall $S_{x}(0)$ with $S_{x}(t) \neq S_{x}(0)$ and $O_{S} \neq 1$. 


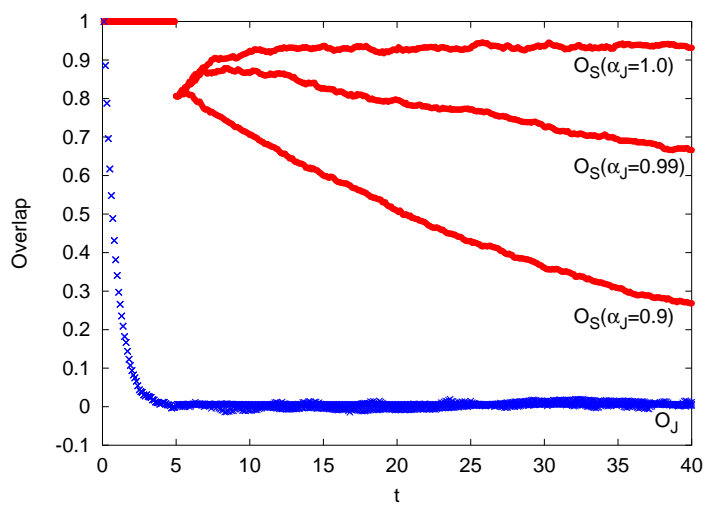

(a) $\beta c_{1}=1.0, c_{2}=c_{3}=0$.

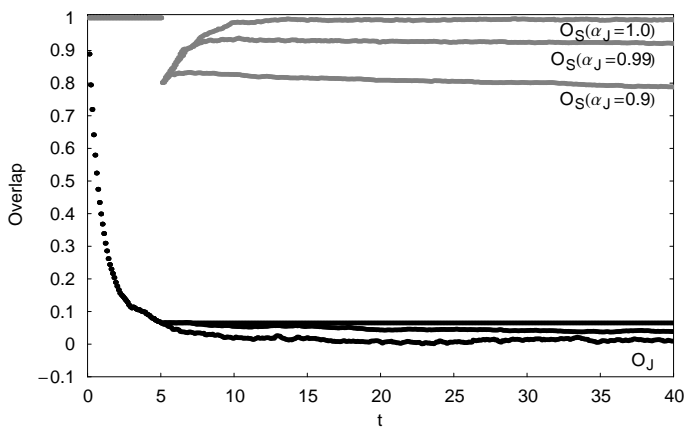

(b) $\beta c_{1}=3.0, \beta c_{2}=-1.0, c_{3}=0$.

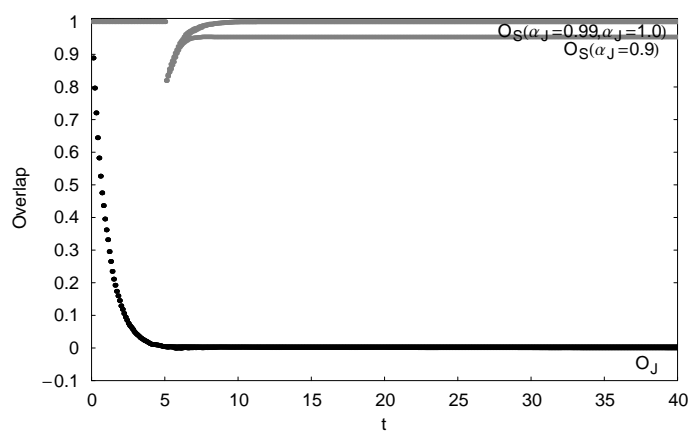

(c) $\beta c_{1}=3.0, \beta c_{2}=1.0, c_{3}=0$.

FIG. 13. $O_{S}(t)$ and $O_{J}(t)$ in simultaneous time variations of $S_{x}$ and $J_{x \mu}$ for $t_{1}<t\left(t_{1}=5\right)$. We choose $\alpha_{J}=0.9$ for $t<t_{1}$, and $\alpha_{S}=0.9, \alpha_{J}=1,0.99,0.9$ for $t_{1}<t$. The point of $c_{i}$ in the case (a) locates near the Higgs-confinement phase boundary in MFT, while the cases (b) and (c) locate in the deep Higgs phase. $O_{J}$ 's for three $\alpha_{J}$ in (a) and (b) are almost degenerate, while $O_{J}$ 's in (b) are for $\alpha_{J}=1,0.99,0.9$ from the above, respectively.

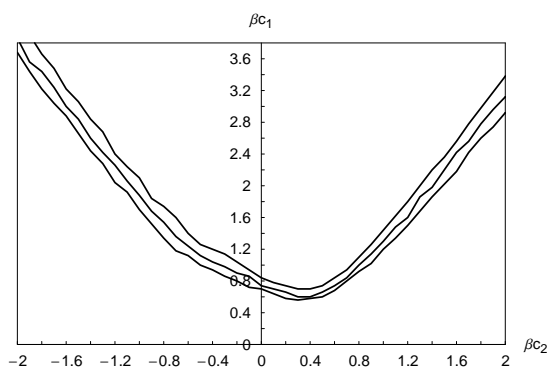

(a) $S_{x}(0)$ random; $J_{x \mu}(0)$ random.

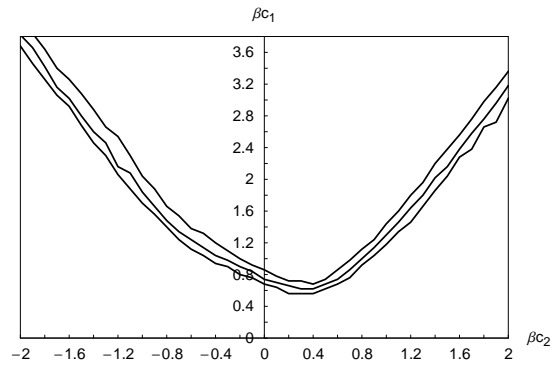

(b) $S_{x}(0)=1 ; J_{x \mu}(0)$ random.

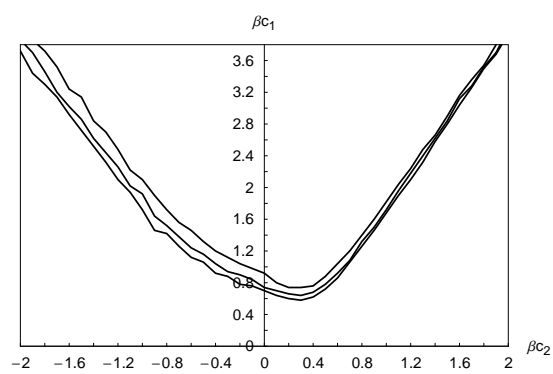

(c) $S_{x}(0)=1 ; J_{x \mu}(0)=-1$

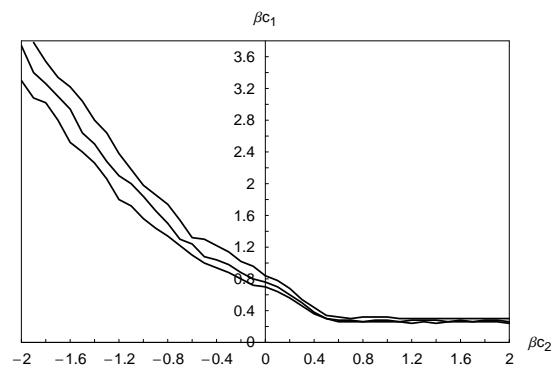

(d) $S_{x}(0)=1 ; J_{x \mu}(0)=1$.

FIG. 14. Contour plots of $O_{s}$ at large $t$ in the $\beta c_{2}-\beta c_{1}$ plane at $c_{3}=0$ for four different initial configurations (a)-(d). Three curves in each figure are contours of $O_{s}=0.9,0.8,0.7$ from the above, respectively. The cases (a),(b),(c) look similarly each other, while the case $(\mathrm{d})$ differs from them. 


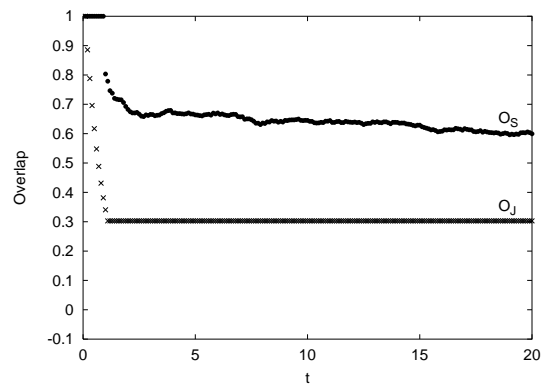

(a)

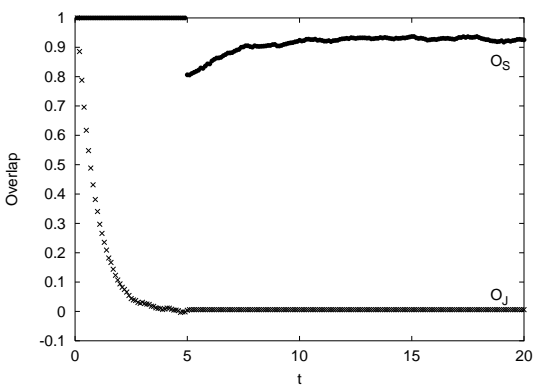

(b)

FIG. 15. Overlaps $O_{S}, O_{J}$ for $\beta c_{1}=1.0, c_{2}=0.0, c_{3}=0.0$ with two different learning times $t_{1}$; (a) $t_{1}=1$ and (b) $t_{1}=5$. The system fails to recall $S_{x}(0)$ in (a) with a small $t_{1}$, while it succeeds in (b) with a suffciently large $t_{1}$.

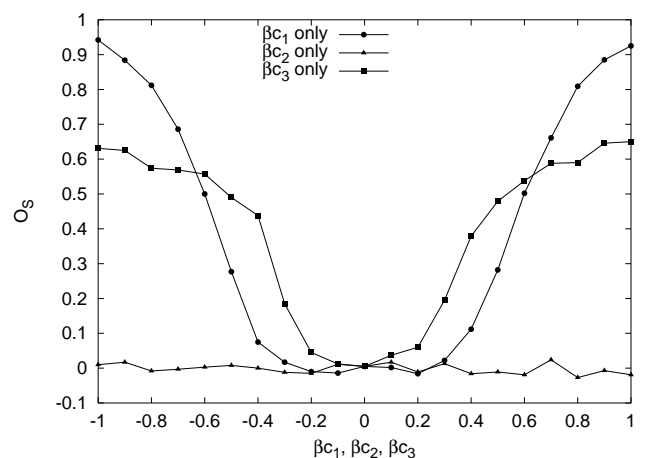

FIG. 16. $O_{s}$ at large $t$ with the three cases; $c_{1}$ only $\left(c_{2}=c_{3}=0\right), c_{2}$ only $\left(c_{1}=c_{3}=0\right)$, and $c_{3}$ only $\left(c_{1}=c_{2}=0\right)$.

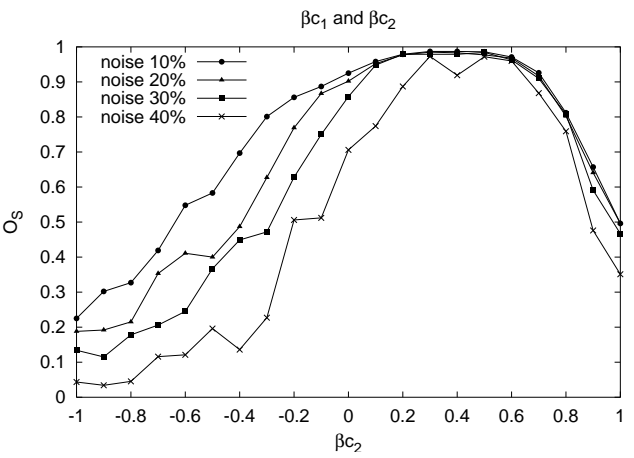

(a)

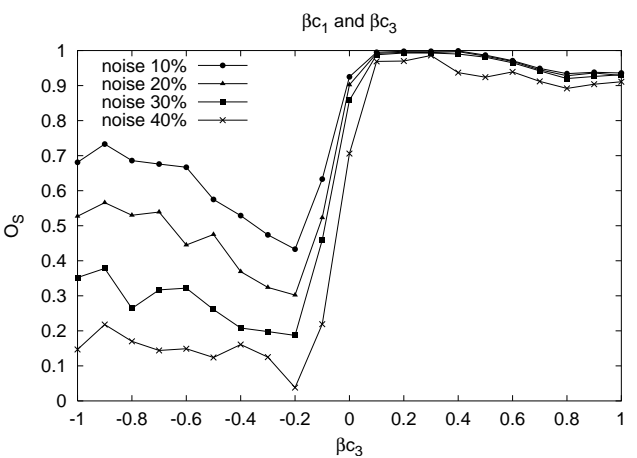

(b)

FIG. 17. $O_{S}$ at sufficiently large time with several $S_{x}\left(t_{1}\right)$ 's produced by adding various amounts of noises upon $S_{x}(0)$. (a) $\beta c_{1}=1.0, c_{3}=0.0$. (b) $\beta c_{1}=1.0, c_{2}=0.0$.

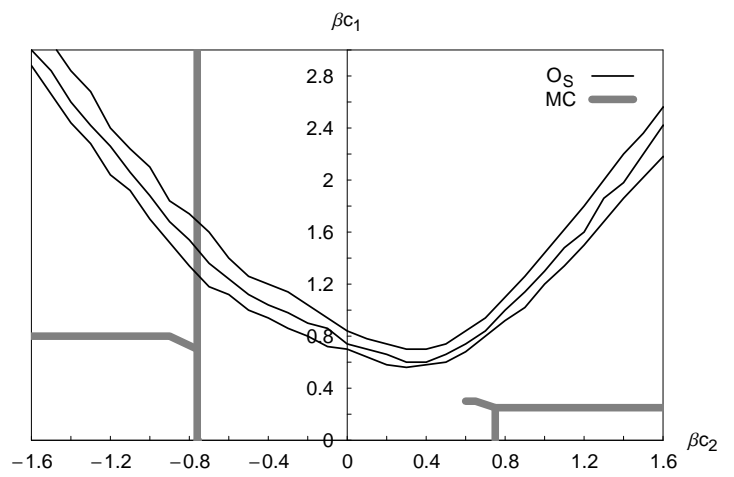

FIG. 18. Contour plot of $O_{S}$ in the $\beta c_{2}-\beta c_{1}$ plane at $c_{3}=0$. Three curves are contours of $O_{s}=0.9,0.8,0.7$ from the above. The phase boundaries of MC simulations in Fig.10 are superposed. 